\title{
Variable Viscosity and Density Biofilm Simulations using an Immersed Boundary Method, Part II: Experimental Validation and the Heterogeneous Rheology-IBM
}

\author{
Jay A. Stotsky ${ }^{\mathrm{a}}$, Jason F. Hammond ${ }^{\mathrm{b}}$, Leonid Pavlovsky ${ }^{\mathrm{c}}$, Elizabeth J. Stewart ${ }^{\mathrm{c}}$, John G. Younger ${ }^{\mathrm{d}}$, Michael J. \\ Solomon ${ }^{\mathrm{c}}$, David M. Bortz ${ }^{\mathrm{a}, *}$ \\ ${ }^{a}$ Department of Applied Mathematics, University of Colorado, Boulder, CO 80309-0526 \\ ${ }^{b}$ High Power Electromagnetic Division, AFRL, Kirtland AFB, NM 87117 \\ ${ }^{c}$ Department of Chemical Engineering, University of Michigan, Ann Arbor, MI 48109 \\ ${ }^{d}$ Department of Emergency Medicine, University of Michigan Ann Arbor, MI 48109
}

\begin{abstract}
The goal of this work is to develop a numerical simulation that accurately captures the biomechanical response of bacterial biofilms and their associated extracellular matrix (ECM). In this, the second of a two-part effort, the primary focus is on formally presenting the heterogeneous rheology Immersed Boundary Method (hrIBM) and validating our model by comparison to experimental results. With this extension of the Immersed Boundary Method (IBM), we use the techniques originally developed in Part I ([19]) to treat biofilms as viscoelastic fluids possessing variable rheological properties anchored to a set of moving locations (i.e., the bacteria locations). In particular, we incorporate spatially continuous variable viscosity and density fields into our model. Although in [14, 15], variable viscosity is used in an IBM context to model discrete viscosity changes across interfaces, to our knowledge this work and Part I are the first to apply the IBM to model a continuously variable viscosity field.

We validate our modeling approach from Part I by comparing dynamic moduli and compliance moduli computed from our model to data from mechanical characterization experiments on Staphylococcus epidermidis biofilms. The experimental setup is described in [26] in which biofilms are grown and tested in a parallel plate rheometer. In order to initialize the positions of bacteria in the biofilm, experimentally obtained three dimensional coordinate data was used. One of the major conclusions of this effort is that that treating the spring-like connections between bacteria as Maxwell or Zener elements provides good agreement with the mechanical characterization data. We also found that initializing the simulations with different coordinate data sets only led to small changes in the mechanical characterization results. Matlab code used to produce results in this paper will be available at https://github.com/MathBioCU/BiofilmSim.
\end{abstract}

Keywords: Navier-Stokes equation, biofilm, immersed boundary method, computational fluid dynamics, viscoelastic fluids

\section{Introduction}

The goal of this work is to show that the model and corresponding simulation method originally developed in part I [19], can accurately capture the biomechanical response of bacterial biofilms. The underlying mathematical technique is an adaptation of the Immersed Boundary Method (IBM) that takes into account the finite volume of bacteria, and the widely variable material parameters anchored to the positions of the bacteria in a biofilm. We call this method the heterogeneous rheology Immersed Boundary Method (hrIBM). With the hrIBM model, biofilms are resolved to a scale at which the contributions of individual bacteria are distinguishable. This allows for detailed modeling of the highly viscous and spatially heterogeneous extracellular matrix (ECM) and, the polysaccharide chains that link bacteria together in biofilms.

A key feature of our simulations is the use of experimental data from live S. epidermidis biofilms to initialize the spatial location of each bacterium. This removes ambiguity about how to represent the biofilm computationally. Motivated by results from [17], we model the spatial heterogeneity of the ECM by treating the ECM as a "polymer brush" with radially dependent viscosity and density fields centered at each bacteria location. In Figure 1, images of the positions, connectivity, and a viscosity isosurface of bacteria in a sample biofilm are shown. When using

\footnotetext{
${ }^{*}$ Corresponding author

Email address: dmbortz@colorado.edu (David M. Bortz)
} 


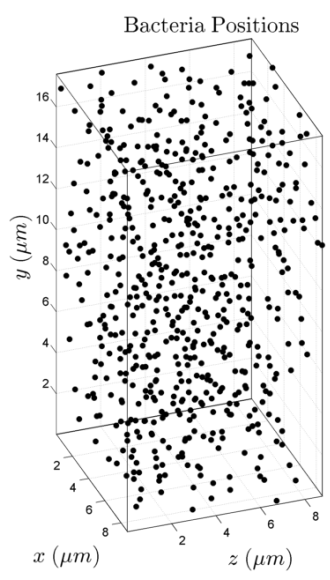

(a)

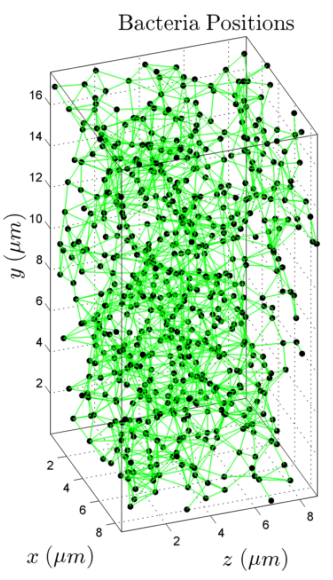

(b)

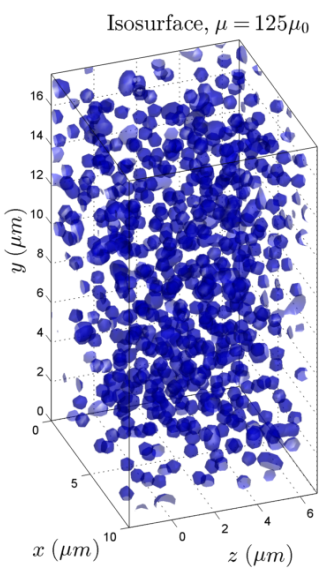

(c)

Figure 1: a) Shows the 3D locations of bacteria from experimental biofilm data. b) Each line represents a viscoelastic connection between two bacteria. Bacteria connected if they are within $1.62 \mu \mathrm{m}$ of each other. c) A viscosity isosurface of the same biofilm. The maximum viscosity is $250 \mu_{0}$ where $\mu_{0}$ is the viscosity of water. The isosurface is the surface defined by $\mu(\mathbf{x})=125 \mu_{0}$.

experimental data to initialize the positions of bacteria, the bulk dynamic moduli and compliance modulus computed through simulation are consistent with experimental results found in [26]. We also show that upon running simulations with several different data sets that possess similar spatial statistics, the physical properties of the biofilm do not change significantly. Finally, we provide quantitative results on the periodic rotation of suspended aggregates of bacteria in shear flow.

In Section 2, we provide a brief review of the classical Immersed Boundary Method (IBM), a well known computational technique for the simulation of coupled fluid-structure interactions. Additionally, we discuss some other IBM-based biofilm models, and explain the adaptations of the IBM that lead to the hrIBM. In Section 3, we give a description of the numerical properties of the hrIBM model and provide results from numerical tests showing that the model is convergent. In Section 4, methods for computing relevant material properties from the hrIBM model are discussed, and the dynamic moduli and compliance moduli computed from the model are compared to experimental data from biofilms grown in a bioreactor. In Sections 5 and 6, we conclude this work and discuss future research directions.

To our knowledge, this work is the first to use a model that accounts for both the heterogeneous rheological properties (e.g., variable viscosity) and, the inter-bacterial connectivity to compute material properties of a biofilm. This work along with Part I, is also the first to incorporate variable viscosity into an IBM-based biofilm model, and the second to use variable viscosity with an IBM model in general. Matlab code used to produce the results obtained in this paper is be available at https://github.com/MathBioCU/BiofilmSim.

\section{The Biofilm Model}

\subsection{Previous IBM Based Biofilm Models}

In recent years, a number of different approaches to IBM-based biological material models have been developed. In [23], an immersed viscoelastic structure is coupled to fluid flow in an immersed boundary type formulation. However, the fluid equations are solved separately from the equations governing the motion of the immersed viscoelastic solid and then coupled together at a sharp physical interface. This strategy works well for systems that have sharp fluid-structure interfaces. We deviate from this strategy since biofilms do not have sharp interfaces, and are permeated by fluid. Thus we invoke a spatially variable viscosity and couple the viscoelastic structure and fluid throughout the entire computational domain.

Another approach to capturing the viscoelastic nature of biofilms with an IBM is through adapting the choice of viscoelastic model governing the forces generated by links between bacteria. This type of strategy was used first by [5] to model general viscoelastic connections in the actin cytoskeleton of ameboid cells. This strategy has also 
been used in [39] to model sperm motility, and in [34] to model immersed three dimensional viscoelastic networks. We pursue this strategy by testing our model with several viscoelastic force laws, and also build upon this class of models with the inclusion of rheologically variable fluid media.

Other IBM-based biofilm models can be found in [2] and in [11]. In these models, an IBM is used directly to couple the forces between connected bacteria with fluid motion. Additionally, some validation results are performed to show that properties such as the recovery and relaxation times of simulated biofilms fall within the range of realistic values. Although not an IBM-based model, we also note that similar results are computed and compared to experiments in [33] where a phase-field model is used. Other phase field models that the reader may find of interest can be found in $[9,35,36,37]$.

In a recent work [32], the IBM is used along with the von Mises stress criterion to provide detailed simulations of the detachment of a biofilm in shear flow. Although we do not currently employ a means of modeling detachment, it is possible to implement a model that allows the breakage of connections between bacteria in the hrIBM framework. If enough connections break, detachment of one or more bacteria from the biofilm can occur. A simple, strain based criterion was used in Part I [19] to model the rupture of links connecting bacteria as deformation occured in a biofilm.

As our biofilm model is based on the IBM, interested readers can find detailed explanations of the IBM in [27, 28, 38, 39], and additional IBM-based biofilm models can be found in [1, 11, 19].

\subsection{The Biofilm Model}

The model we use is comprised of two sets of equations; those that model fluid flow through the biofilm, and those that model motions and forces experienced by each bacteria cell in the biofilm. These equations are listed in (1)-(8).

$$
\begin{array}{r}
\rho(\mathbf{x}, t)\left(\mathbf{u}_{t}+(\mathbf{u} \cdot \nabla) \mathbf{u}\right)=-\nabla P+\nabla \cdot\left(\mu(\mathbf{x}, t)\left(\nabla \mathbf{u}+(\nabla \mathbf{u})^{T}\right)\right)+\mathbf{f}(\mathbf{x}, t) \\
\nabla \cdot \mathbf{u}=0 \\
\mathbf{U}(s, t)=\int_{\Omega} \mathbf{u}(\mathbf{x}, t) \hat{\delta}(\mathbf{X}(s, t)-\mathbf{x}, \omega) d \mathbf{x} s=1,2, \ldots N \\
\frac{\partial \mathbf{X}(s, t)}{\partial t}=\mathbf{U}(s, t) \\
\mathbf{F}(s, t)=\mathcal{F}(\mathbf{X}(s, t), \mathcal{P}) \\
\mathbf{f}(\mathbf{x}, t)=\frac{1}{d_{0}^{3}} \sum_{s=1}^{N} \mathbf{F}(s, t) \hat{\delta}(\mathbf{X}(s, t)-\mathbf{x}, \omega) \\
\rho(\mathbf{x}, t)=\rho_{0}+(2 \omega)^{3} \sum_{s=1}^{N}\left(\rho_{b}-\rho_{0}\right) \hat{\delta}(\mathbf{X}(s, t)-\mathbf{x}, \omega) \\
\mu(\mathbf{x}, t)=\mu_{0}+(2 \omega)^{3} \max _{1 \leq s \leq N}\left(\mu_{b}-\mu_{0}\right) \hat{\delta}(\mathbf{X}(s, t)-\mathbf{x}, \omega)
\end{array}
$$

These equations are similar to those used in Part I [19] except for some small changes that were convenient for implementing the simulations described in Section 4.

In most IBM literature, the Equations 6 and 7 are written as integrals over Lagrangian coordinates. In this case, there is a fixed, finite number of bacteria in the domain regardless of refinement level, so we compute these as summations instead. In these equations, we use a scaled approximation of the Dirac $\delta$-function, denoted $\hat{\delta}$, which has a region of support that depends on a radial parameter $\omega$, defined such that $2 \omega$ is the hydrodynamic radius of a typical bacteria. Since only finitely many bacteria are considered, we also define a Lagrangian label $s$ as a number associated with each bacteria.

The scale factor of $(2 \omega)^{3}$ in Equations 7 and 8 is chosen to enforce that at the locations, $\mathbf{X}(s, t)$, the density and viscosity are equal to $\rho_{b}$ and $\mu_{b}$ respectively. Finally, we define $\mathbf{F}$ as a force instead of a force density to allow for standard units to be associated with the constitutive parameters (i.e. spring constants) that will be used to model the viscoelastic links between neighboring bacteria. Complete listings of the quantities appearing in the model equations can be found in Tables 1, 2, and 3 .

Since individual bacteria are not assumed to have infinitessimal volume at the scale of our simulations, the Lagrangian quantities; $\mathbf{X}, \mathbf{U}, \mu_{b}, \rho_{b}$, and $\mathbf{F}$ correspond to measurements taken at the center of mass of each bacterium. As described in Section 2.3, the second argument, $\omega$, of the smoothed Dirac $\delta$ function, $\hat{\delta}(\cdot, \omega)$ determines

a region of support for the smoothed $\delta$ function. The choice of $\hat{\delta}(\cdot, \omega)$ govern how the mass density, viscosity, and force density vary around each bacterium. 


\begin{tabular}{|c|c|}
\hline \multicolumn{2}{|c|}{ Eulerian Variables } \\
\hline \hline Symbol & Definition \\
\hline $\mathbf{x}$ & Eulerian position \\
\hline$t$ & time \\
\hline $\mathbf{u}$ & Eulerian velocity \\
\hline$P$ & pressure \\
\hline $\mathbf{f}(\mathbf{x}, t)$ & Eulerian force density \\
\hline$\mu(\mathbf{x}, t)$ & Eulerian viscosity \\
\hline$\rho(\mathbf{x}, t)$ & Eulerian density \\
\hline
\end{tabular}

Table 1: Quantities assigned a value at each grid point and timestep in the discretized computational domain. Together they provide an Eulerian description of the fluid motion in the biofilm.

\begin{tabular}{|c|c|}
\hline \multicolumn{2}{|c|}{$\begin{array}{c}\text { Quantities Associated with each Bacterium } \\
\text { (Lagrangian variables) }\end{array}$} \\
\hline \hline Symbol & Definition \\
\hline$s$ & label for each bacteria \\
\hline $\mathbf{X}(s, t)$ & center of mass of bacteria $s$ at time $t$ \\
\hline $\mathbf{U}(s, t)$ & velocity of bacteria $s$ at time $t$ \\
\hline $\mathbf{F}(s, t)$ & force on bacteria $s$ at time $t$ \\
\hline$\mu_{b}$ & viscosity at bacteria center of mass \\
\hline$\rho_{b}$ & density at bacteria center of mass \\
\hline
\end{tabular}

Table 2: Quantities assigned to each bacteria in the domain at each time step. They can be thought of as Lagrangian variables associated with each bacteria center of mass.

\begin{tabular}{|c|c|}
\hline \multicolumn{2}{|c|}{ Model Parameters } \\
\hline \hline Symbol & Definition \\
\hline$\mu_{0}$ & viscosity of water \\
\hline$\rho_{0}$ & density of water \\
\hline $\mathcal{F}(\mathbf{X}, \mathcal{P})$ & constitutive relation between bacteria configuration and force \\
\hline $\mathcal{P}$ & parameters in the viscoelastic law used (i.e. spring coefficients etc.) \\
\hline$N$ & number of bacteria in computational domain \\
\hline$d_{0}^{3}$ & volume of average bacterium \\
\hline$\omega$ & hydrodynamic radius of a bacterium \\
\hline$r_{c}$ & cutoff radius for establishing which bacteria are linked \\
\hline$\hat{\delta}(\cdot, \omega)$ & discrete Dirac delta function with support related to parameter $\omega$ \\
\hline
\end{tabular}

Table 3: These quantities are parameters to the model. They are either based on physical data (i.e. density and viscosity of water) or are tuned through simulations to obtain realistic values. 
The viscosity is computed using a different form than the density and force and is shown in Equation (8). This formula for viscosity is not intuitive, and an explanation for this choice of viscosity can be found in Part I. However, finding the most accurate form for $\mu(\mathbf{x})$ is still an area of active research. In particular, we experimented with approximations to $\mu(\mathbf{x})=\mu_{0}+\int_{\Omega}\left(\mu_{b}-\mu_{0}\right) \hat{\delta}(\mathbf{X}(t)-\mathbf{x}, \omega) d \mathbf{X}$. We made our ultimate choice for $\mu(\mathbf{x})$ so that the viscosity does not overshoot $\mu_{b}$, the force and viscosity drop off at the same rate, and to maintain consistency with Part I.

The last part of the model is a force law, $\mathcal{F}$ needed to specify $\mathbf{F}$ based on the configuration of bacteria, $\mathbf{X}$. We use Hooke's law, Maxwell's law, and the Zener model in our simulations, and initialize the connectivity of the biofilm by using a cutoff radius, $r_{c}$. At the start of the simulation, any two bacteria that are separated by a distance less than the cutoff radius will be linked by a spring whose initial, resting length is their initial separation.

By using the IBM as a basis for our biofilm model, we avoid treating the biofilm as a two phase fluid with a distinct bulk fluid region and a distinct biofilm region. Instead, the use of variable rheological properties over the entire domain couples the biofilm and bulk fluid motions as a single viscoelastic material. Because the fluid permeates through the entire domain, even when the simplest force law, Hooke's Law, is used to determine forces between bacteria, the model still behaves viscoelastically, not elastically.

\subsection{The Heterogeneous Rheology Immersed Boundary Method}

In our model, we extend the IBM to account for the fixed, finite size of bacteria and allow for variable physical properties that are anchored to a moving Lagrangian mesh (i.e. the bacteria positions). We denote this approach the heterogeneous rheology IBM (hrIBM).

The original IBM was first developed as a means of solving fluid-structure interaction problems in cardiology and is applicable to problems with moving, irregularly shaped boundaries [27, 28]. With the IBM, the fluid velocity fields and pressure are usually solved for on a fixed, Eulerian grid and the movement of the boundaries due to fluid motion is tracked by a Lagrangian mesh that moves according to the underlying fluid motion. As the material boundaries are deformed, a constitutive model is used to determine the force density exerted by the immersed boundary on the fluid. The Lagrangian force density field is then transferred to an Eulerian force density field through a convolution integral with a $\delta$ function kernel. The Eulerian force density influences the fluid motion through its inclusion in the Navier-Stokes equations. Thus the fluid and boundary motions are coupled since the boundary motion is interpolated from the fluid velocity field, and the fluid velocity field is influenced by forces on the boundary. The couplings and solution process for the IBM are depicted in Figure 2.

\subsubsection{A Dirac $\delta$ Approximation Based on Finite Volume Bacteria}

An important step in the numerical implementation of the IBM is the construction of a discrete approximation of the Dirac $\delta$ function (denoted $\hat{\delta}(\cdot, h)$ ). This approximation is chosen such that as $h \rightarrow 0, \hat{\delta}(\mathbf{r}, h) \rightarrow \delta(\mathbf{r})$. This is accurate for fluid structure interactions involving fluid-solid boundaries that have infinitessimal thickness, and thus zero volume. In the hrIBM model, each Lagrangian point corresponds to the center of mass of a bacterium which has a finite volume. Therefore, we use a smoothed version of the standard discrete $\delta$ function whose region of support is controlled by a radial parameter, $\omega$, that is independent of the grid spacing, $h$.

In our model, we use a smoothed discrete Dirac $\delta$ approximation of the form,

$$
\hat{\delta}(\mathbf{x}, \omega)=\frac{1}{\omega^{3}} \phi\left(\frac{x}{\omega}\right) \phi\left(\frac{y}{\omega}\right) \phi\left(\frac{z}{\omega}\right)
$$

with $\phi(r)$ as defined in [28] by

$$
\phi(r)=\left\{\begin{array}{cc}
\frac{1}{8}\left(5-2|r|-\sqrt{-7+12|r|-4|r|^{2}}\right) & 1 \leq|r| \leq 2 \\
\frac{1}{8}\left(3-2|r|+\sqrt{1+4|r|-4|r|^{2}}\right) & 0 \leq|r| \leq 1 \\
0 & |r|>2
\end{array}\right.
$$

Further discussion of this choice for $\hat{\delta}(\mathbf{x}, \omega)$ can be found in [19]. If $\omega=h$, the standard discrete $\delta$ function seen in IBM literature is obtained. For this work, we assume that the bacteria are spherical and thus $\omega$ is understood as a hydrodynamic radius. We also note that extensions to this formalism will allow for the treatment of nonspherical bacteria or unevenly sized bacteria. Thus, $\omega$ may be though of more generally as a shape parameter. 


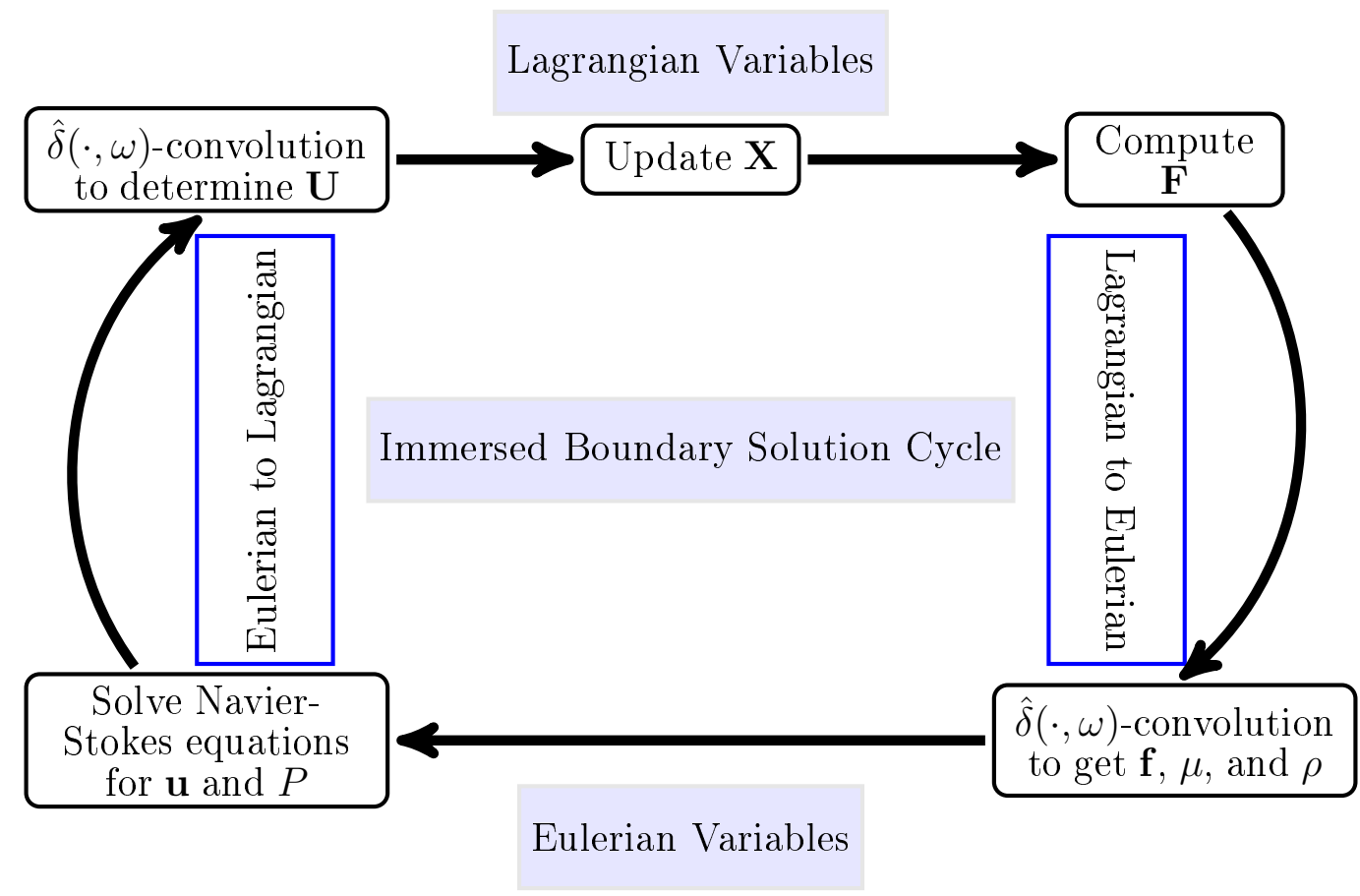

Figure 2: The coupling between the Eulerian and Lagrangian variables in the hrIBM is shown here. The Eulerian and Lagrangian variables are coupled by the computation of $\mathbf{U}$ from $\mathbf{u}$, and the computation of $\mathbf{f}, \mu$, and $\rho$ from $\mathbf{F}$ and $\mathbf{X}$. The IBM is a widely applicable method in part because it allows for a great variety of fluid solvers and solid structural models to be coupled through $\delta$ function transfer identities.

\subsubsection{Variable Viscosity and Density in the hrIBM}

Highly heterogeneous viscosity and moderately heterogeneous density are common characteristics of biofilms. Although IB methods with variable density have existed for some time (see [38]), the incorporation of spatially variable viscosity in the IBM is an area that has yet to be well developed. We note that in [14] and [15], an IBM capable of solving problems with variable viscosity and density is used to model the motion of red blood cells flowing in capillaries. When modeling red blood cells, the viscosity exhibits a "jump" discontinuity between the blood plasma and the intracellular, hemoglobin-containing fluid of a red blood cell. Thus, their model is designed to capture the dynamics of two interacting fluids with different rheological properties separated by a deformable membrane. In our case, there do not exist well defined boundaries and thus, $\delta(\cdot, \omega)$ is adjusted to reflect this.

In biofilms, the spatial variance of material properties is localized around the position of each bacterium, while in fluid far away from any bacteria, the physical properties are those of the bulk fluid. This localization of the variation in material properties allows the spatial variation in density and viscosity to be found by using a smoothed $\delta$-function integration similar to that used to compute the Eulerian force field. We define an viscosity, $\mu_{b}$ and density, $\rho_{b}$ and assume that at the center of mass of each bacteria, the viscosity and density are $\rho\left(\mathbf{X}_{i}, t\right)=\rho_{b}$, and $\mu\left(\mathbf{X}_{i}, t\right)=\mu_{b}$. Defining $\mu_{0}$ to be the viscosity of the bulk fluid (in this case water) the viscosity at any Eulerian grid point is calculated as:

$$
\mu(\mathbf{x})=\mu_{0}+(2 \omega)^{3} \max _{1 \leq s \leq N}\left[\left(\mu_{b}-\mu_{0}\right) \hat{\delta}(\mathbf{x}-\mathbf{X}(s), \omega)\right] .
$$

The density can be computed from

$$
\rho(\mathbf{x}, t)=\rho_{0}+(2 \omega)^{3} \sum_{s=1}^{N}\left(\rho_{b}-\rho_{0}\right) \hat{\delta}(\mathbf{X}(s, t)-\mathbf{x}, \omega) .
$$

In the simulations, the number of bacteria, $N$, is fixed and independent of the mesh spacing $h$, thus a summation is used instead of an integral for the density computation.

In this model, we indirectly take into account the fluid volume displacement caused by the presence of the bacteria. We treat the localized high viscosity around each bacteria as an effective viscosity that accounts for both the displaced fluid volume and the increased viscosity near the bacteria surface [17]. Extensions based on changing our choice for $\hat{\delta}(\mathbf{x}, \omega)$ and $\mu(\mathbf{x})$ could possibly allow for a more precise computation of the volume displacement 
caused by the biofilm into the model. In Figure 3, observe that lower strain rates are found near the bacteria cells where high viscosity is present. This is indicative of the bacteria appearing as relatively solid objects compared to the bulk fluid.

We make the assumption that the bacteria have a uniform diameter of $1.0 \mu \mathrm{m}$ based on [18] and [31] where bacteria diameters are measured and determined to have a tightly centered distributions around $0.64 \mu \mathrm{m} 0.70 \mu \mathrm{m}$ respectively. We choose a larger radius since we want to account for the influence of the polymeric ECM which extends into the fluid outside the cell. We also note another source that states that $S$. epidermidis typically have a diameter in the range of $0.5-1.0 \mu \mathrm{m}$ [16]. We also assume that the bacteria are uniformly spherical in shape. Although we have not been able to find data to quantify this assumption, examination of high-resolution images of S. epidermidis bacteria found in [30] and [25] appears to confirm the validity of this assumption.

\section{Numerical Methods}

The numerical methods we use are based on those originally discussed in [19]. We summarize them here for convenience and also provide convergence results. To approximate solutions to equations (1)-(8), we use an approximate projection method similar to that used in [38]. The solution scheme uses an implicit Euler solver to update an intermediate velocity profile at each time step and is expected to be $\mathcal{O}(\Delta t)$ convergent in the velocity field. To discretize the domain, we use a uniform finite difference discretization with equal spacings in the $x, y$, and $z$ directions. The spatial derivatives are approximated with 2 nd order, centered finite differences.

\subsection{Numerical Algorithm}

At each time step, the following quantities must be updated: $\mathbf{u}, \mathbf{U}, P, \mathbf{F}, \mathbf{f}, \mathbf{X}, \mu$, and $\rho$. We nondimensionalize the problem with the following choices:

$$
\hat{\mathbf{u}}=\frac{\mathbf{u}}{u_{0}} \quad \hat{P}=\frac{P}{\rho_{0} u_{0}^{2}} \quad \hat{\mathbf{f}}=\frac{L \mathbf{f}}{\rho_{0} u_{0}^{2}} \quad \hat{\mu}=\frac{\mu}{\mu_{0}} \quad \hat{\rho}=\frac{\rho}{\rho_{0}} \quad \hat{\mathbf{x}}=\frac{\mathbf{x}}{L} \quad \hat{t}=\frac{t}{t_{0}}
$$

and also introduce the following nondimensional parameters:

$$
R e=\frac{\rho_{0} L u_{0}}{\mu_{0}} \quad S t=\frac{L}{t_{0} u_{0}} .
$$

As is standard terminology, Re is the Reynold's number and $S t$ is the Strouhal number. Additionally, we define $d_{0}^{3}$ to be the average Lagrangian volume element as described in Part I [19]. For convenience, we will now assume that all quantities are nondimensional unless otherwise stated. The values of the constants we use are listed in Table 4 and the motivation for these values is discussed in Part I.

As is standard practice in IBM algorithms, we uncouple the Eulerian variable updates and Lagrangian variable updates for computational reasons. At each time step, we use a projection-based solver to solve the Navier-Stokes equation for $\mathbf{u}$ and $P$. We define $\mathbf{G}_{h}$ a discrete gradient operator, and $\mathbf{D}_{h}$ a discrete divergence operator, and use the following approximate projection method to obtain $\mathbf{u}$ and $P:^{1}$

1. Solve for $\mathbf{u}^{*}$

$$
\begin{aligned}
\rho^{(n)}\left(S t \frac{\mathbf{u}^{*}-\mathbf{u}^{(n)}}{\Delta t}+\frac{1}{2}\left(\mathbf{u}^{(n)} \cdot\right.\right. & \left.\left.\mathbf{D}_{h}\left(\mathbf{u}^{(n)}\right)+\mathbf{D}_{h}\left(\mathbf{u}^{(n)} \mathbf{u}^{(n)}\right)\right)\right) \\
& =\frac{1}{\operatorname{Re}} \mathbf{D}_{h}\left(\mu^{(n)}\left(\mathbf{G}_{h}\left(\mathbf{u}^{*}\right)+\left(\mathbf{G}_{h}\left(\mathbf{u}^{*}\right)\right)^{T}\right)\right)+\mathbf{f}^{(n)}
\end{aligned}
$$

2. Solve for $P^{(n)}$

$$
\mathbf{D}_{h}\left(\frac{1}{\rho^{(n)}} \mathbf{G}_{h} P^{(n)}\right)=S t \frac{\mathbf{D}_{h}\left(\mathbf{u}^{*}\right)}{\Delta t}
$$

3. Compute $\mathbf{u}^{(n+1)}$

$$
\mathbf{u}^{(n+1)}=\mathbf{u}^{*}-\left(\frac{1}{S t}\right) \frac{\Delta t}{\rho^{(n)}} \mathbf{G}_{h}\left(P^{(n)}\right)
$$

\footnotetext{
${ }^{1}$ Numerically, products of the form, $\mathbf{D}_{h} a^{(n)} \mathbf{G}_{h}$ are discretized using standard finite difference stencils rather than as a product of the gradient and divergence operators.
} 


\begin{tabular}{|c|c|c|}
\hline Quantity & Value & Explanation \\
\hline \hline$\mu_{0}$ & $1 \cdot 10^{-3} \mathrm{~Pa} \cdot \mathrm{s}$ & viscosity of water at $20^{\circ} \mathrm{C}$ \\
\hline$\rho_{0}$ & $998 \mathrm{~kg} / \mathrm{m}^{3}$ & density of water at $20^{\circ} \mathrm{C}$ \\
\hline$L$ & $10 \mu \mathrm{m}$ & characteristic length scale \\
\hline$R e$ & $0.0014-0.0175$ & Strouhal number \\
\hline$S t$ & $0.0075-0.0720$ & characteristic time \\
\hline$t_{0}$ & $1 \mathrm{~s}$ & Reynold's number. Varies based on the simulation \\
\hline$u_{0}$ & $1.40 \cdot 10^{-4}-0.0017 \mathrm{~m} / \mathrm{s}$ & characteristic velocity, chosen to match experimental strain conditions \\
\hline$d_{0}$ & $1.59 \cdot 10^{-6} \mathrm{~m}$ & average volume of each bacterium, \\
& $0.12 \rho_{0}$ & added density contribution from ECM diver bacteria \\
\hline$\rho_{b}$ & $450 \mu_{0}$ & added viscosity from ECM \\
\hline$\mu_{b}$ & $1.64 \cdot 10^{-11} \mathrm{~N}$ & Hooke's law force constant \\
\hline$F_{s 1}$ & $1.62 \cdot 10^{-6} \mathrm{~m}$ & maximum distance by which bacteria can be connected $t=0$ \\
\hline$r_{c}$ & $2.5 \cdot 10^{-7} \mathrm{~m}$ & scaling constant, equal to $1 / 2$ the hydrodynamic radius of a bacteria \\
\hline$\omega$ & $5.6 \cdot 10^{-12} \mathrm{~N} / \mathrm{s}$ & damping coefficient in Maxwell and Zener models \\
\hline$F_{d 1}$ & $1.64 \cdot 10^{-11} \mathrm{~N}$ & second force constant used in Zener model \\
\hline$F_{s 2}$ & &
\end{tabular}

Table 4: Values of Physical Parameters and Nondimensional constants used in simulations. The values of $F_{s 1}, F_{s 2}$, and $F_{d 1}$ vary depending on whether Hooke's Law, the Maxwell Model, or the Zener Model are used, so the values shown here are for the Zener model which appeared to perform most favorably compared to experimental results. The coefficients for the other two models are discussed in Section 4.2 .

In steps 1 and 2, full multigrid solvers and multigrid preconditioned conjugate gradient solvers are used to find $\mathbf{u}^{*}$ and $P^{(n) 2}$. After obtaining the updated velocity and pressure, the Lagrangian velocity and position updates follow,

$$
\begin{gathered}
\mathbf{U}^{(n+1)}=\sum_{h \in \mathcal{G}_{h}} \mathbf{u}^{(n+1)} \hat{\delta}\left(\mathbf{x}_{h}-\mathbf{X}^{(n)}, h\right) h^{3} \\
\mathbf{X}^{(n)}=\mathbf{X}^{(n-1)}+\frac{\Delta t}{S t} \mathbf{U}^{(n)} .
\end{gathered}
$$

Next the Lagrangian force density is computed based on the new positions, $\mathbf{X}^{(n+1)}$ as $\mathbf{F}^{(n+1)}=\mathcal{F}\left(\mathbf{X}^{(n+1)}\right)($ see section 4.2 for more details). Finally, the Eulerian fields, $\mathbf{f}$ and $\rho$ are computed using discrete $\delta$ function interpolation to the Eulerian grid through equations of the form:

$$
\begin{aligned}
\mathbf{f}^{(n+1)} & =\sum_{s=1}^{N}\left(\mathbf{F}^{(n+1)}(s) / d_{0}^{3}\right) \hat{\delta}\left(\mathbf{x}_{h}-\mathbf{X}^{(n+1)}(s), \omega\right) d_{0}^{3} \\
\rho^{(n+1)} & =\rho_{0}+(2 \omega)^{3}\left(\rho_{b}-\rho_{0}\right) \sum_{s=1}^{N} \hat{\delta}\left(\mathbf{x}_{h}-\mathbf{X}^{(n+1)}(s), \omega\right),
\end{aligned}
$$

and $\mu$ is computed from

$$
\mu^{(n+1)}=\mu_{0}+(2 \omega)^{3} \max _{1 \leq s \leq N}\left[\left(\mu_{b}-\mu_{0}\right) \hat{\delta}\left(\mathbf{x}-\mathbf{X}^{(n+1)}(s), \omega\right)\right]
$$

In the simulations we conduct, the primary direction of fluid flow is in the $z$ direction. The height is governed by the $y$ coordinate and width by the $x$ coordinate. In Figure 5, the motion of the bacteria in a simulation with these conditions is depicted.

\subsection{Numerical Verification and Convergence Properties}

In the first numerical verification result, we verify the accuracy of the numerical projection method solver with no biofilm present by comparing the numerical solution with an analytical solution. Since there is no immersed

\footnotetext{
${ }^{2}$ Strictly speaking, the pressure should be interpreted as being defined at the half-integer time steps as discussed in [6]. We use the notation $P^{(n)}$ for simplicity
} 


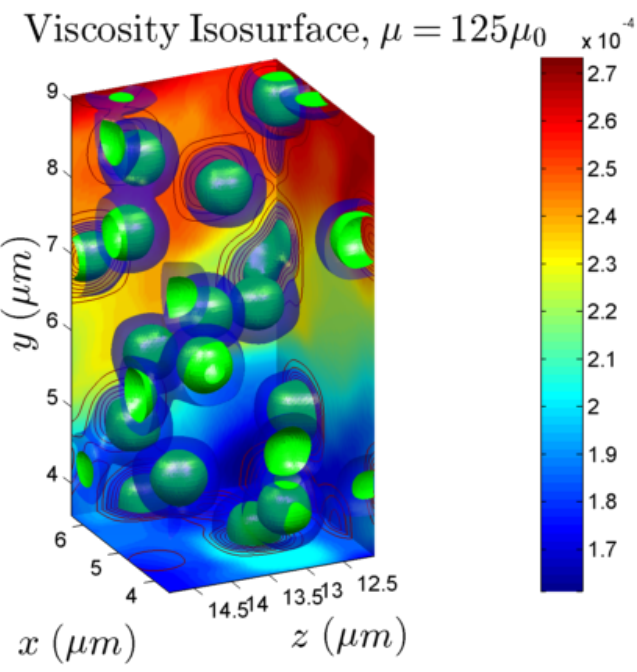

(a)

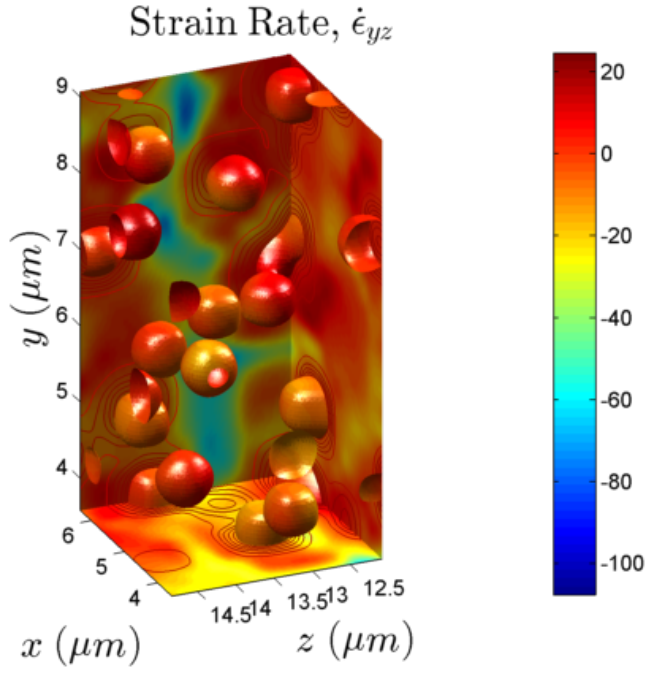

(b)

Figure 3: a) A viscosity isosurface is shown for a small section of a biofilm used in simulation. The inner isosurface is $\mu=125 \mu_{0}$ and the outer transparent isosurface is at $\mu=50 \mu_{0}$. Slices of the $\|\mathbf{u}\|$ velocity field are shown as well. b) The $\dot{\epsilon}_{y z}$ component of the strain rate is plotted on the $\mu=125 \mu_{0}$ viscosity isosurface. Additionally, the strain rate and contours of viscosity are shown in slice planes. For a single phase fluid, Newton's viscosity law is $\sigma=\mu \dot{\epsilon}$. Although biofilms are not Newtonian fluids, we still see that in areas of low viscosity, higher strain rates are found and in areas of higher viscosity lower strain rates occur.

\begin{tabular}{|c|c|c|c|}
\hline Frequency of Boundary Oscillation & Time & Space & Error $\left\|\mathbf{u}^{h}-\mathbf{u}\right\|_{2}$ \\
\hline $49.91 \mathrm{~Hz}$ & 1.735 & 1.6928 & $1.8363 \cdot 10^{-5}$ \\
\hline $499.1 \mathrm{~Hz}$ & 1.246 & 1.9817 & $5.6811 \cdot 10^{-5}$ \\
\hline
\end{tabular}

Table 5: Average convergence factors of the Fluid Solver. Spatial convergence tests were carried out with grid spacings, $h$, set to $1 / 32$, $1 / 64$, and $1 / 128$ and a time step of $\Delta t=1 / 2500 / \nu$. Temporal convergence tests were done with $\nu \Delta t$ set to $\pi / 125, \pi / 250$, and $\pi / 500$ and $h=1 / 32$. Error is computed at $t=(0.5 \pi / \nu) s$ for temporal convergence tests and after 1250 time steps for the spatial convergence tests. Convergence factors are computed as $\rho(\Delta t)=\log _{2}\left(\frac{\left\|u(\Delta t)-u_{\text {exact }}\right\|_{2}}{\left\|u(\Delta t / 2)-u_{\text {exact }}\right\|_{2}}\right)$ in time and by $\rho(h)=\log _{2}\left(\frac{\left\|u(h)-u_{\text {exact }}(h)\right\|_{2}}{\left\|u(h / 2)-u_{\text {exact }}(h / 2)\right\| \|_{2}}\right)$ in space and $u_{\text {exact }}(h)$ is the exact solution on a grid with spacing $h$. For the spatial convergence tests, we found it necessary to increase the Reynold's number by a factor of 1000 to ensure that the error in the iterative solver and the temporal discretization was much smaller than the spatial discretization error. Before this change the discretization error was only $\mathcal{O}\left(10^{-9}\right)$.

structure, this is a test of the fluid solver alone, and not the IBM method. For this test, the domain, $\Omega$ is chosen to be a rectangular solid that is periodic in the $x$ and $z$ directions. From [8], the following boundary conditions for $y=0$ and $y_{L}$,

$$
\frac{\partial P}{\partial y}=\left.0 \quad \mathbf{u}\right|_{0}=\left.(0,0,0) \quad \mathbf{u}\right|_{y_{L}}=(0,0, \sin \nu t)
$$

provide us with an analytic solution,

$$
u_{z}(y, t)=\left|\frac{\sinh k y(1+i)}{\sinh k y_{L}(1+i)}\right| \sin \left(\nu t+\arg \left(\frac{\sinh k y(1+i)}{\sinh k y_{L}(1+i)}\right)\right) \quad k=\left(\frac{\nu \rho}{2 \mu}\right)^{1 / 2} .
$$

The values of $P, u_{x}$ and $u_{y}$ are exactly zero in this case. The values of $\rho$ and $\mu$ are set to $998 \mathrm{~kg} / \mathrm{m}^{3}$ and $1 \mathrm{mPa} \cdot s$ respectively and are homogenous across the domain since no analytic solutions with variable density and viscosity and the boundary conditions given above are known to the authors. Convergence tests were conducted with frequencies $\nu=4.991 \mathrm{~Hz}, 49.91 \mathrm{~Hz}$ with the biofilm, and at $49.91 \mathrm{~Hz}$ and $499.1 \mathrm{~Hz}$ without the biofilm. In Table 5 the absolute error, temporal convergence factors, and spatial convergence factors are listed. For a full explanation and justification of these tables, see Part I [19].

Additionally, with the same boundary conditions as above, we tested the convergence rates for simulations with a biofilm that possesses variable density and viscosity. Temporal and spatial convergence factors are shown in Table 6. As is often seen with immersed boundary methods, discretization error induced by the presence of discrete $\delta$ functions, in the transferrence of density, viscosity, and forces between Eulerian and Lagrangian coordinates leads to a reduction to first order convergence $[28,20]$. In some cases, second order accuracy can be recovered, however 


\begin{tabular}{|c|c|c|c|c|}
\hline \multirow{2}{*}{ Frequency of Boundary Oscillation } & \multicolumn{2}{|c|}{ Velocity, $\|\mathbf{u}\|$} & \multicolumn{2}{c|}{ Position, $\|\mathbf{X}\|$} \\
\cline { 2 - 5 } & Time & Space & Time & Space \\
\hline $49.91 \mathrm{~Hz}$ & 0.983 & 1.105 & 1.022 & 0.952 \\
\hline $4.991 \mathrm{~Hz}$ & 0.991 & 0.910 & 1.007 & 1.054 \\
\hline
\end{tabular}

Table 6: Convergence factors of hrIBM with biofilm. For spatial convergence, $h$ was set to $1 / 32,1 / 64$, and $1 / 128$ with a time step of $\nu \Delta t=$ $1 / 500$. To measure the temporal convergence factors, $\nu \Delta t$ was set to $1 / 250,1 / 500$, and $1 / 1000$. In both cases, the boundary conditions described in Section 3 were used. Note, that $\nu$ refers to oscillation frequency, not dynamic viscosity. Temporal convergence factors were computed as $\rho(\Delta t)=\log \frac{\|u(\Delta t)-u(\Delta t / 2)\|}{\|u(\Delta t / 2)-u(\Delta t / 4)\|}$ and spatial convergence factors were computed as $\rho(\Delta x)=\log \frac{\left\|u(\Delta x)-I_{h / 2}^{h} u(\Delta x / 2)\right\|}{\left\|u(\Delta x / 2)-I_{h / 4}^{h / 2} u(\Delta x / 4)\right\|}$ where $I_{h}^{2 h}$ is an interpolation operator from a grid of spacing $h$ to a grid of spacing $2 h$. For spatial refinement, we also note that the number of Lagrangian nodes (bacteria) remains constant as we are not approximating a surface, but instead treating each Lagrangian node as a single bacteria which should have a constant volume independent of the grid refinement.

this generally requires predictor-corrector methods that are more computationally expensive than what we use here. In addition, variable density tends to lead to first order in time methods even when the velocity is discretized by a formally second order accurate scheme $[14,15]$. More detailed numerical convergence results for this model with different boundary conditions are shown in [19]. In Table 6, temporal convergence factors for the same fluid conditions and domain as the analytical solution are listed. The pressure convergence is not shown here since we use a "pressure-free" projection method in our simulations (see [6] for more details), and because the effect of pressure on the velocity field is several orders of magnitude less than the viscous and elastic effects on the scale of our simulations.

\section{Experimental Validation Results}

The material characterization of bacterial biofilms is a difficult experimental task. It is usually not possible to grow biofilms large enough for use in standard testing devices and, attempts to move a biofilm from the environment it was grown in to a testing apparatus may alter its structure. In [26], a promising experimental method of testing material properties of biofilms was developed. In the experimental setup, a biofilm is grown in a parallel plate rheometer that is $40 \mathrm{~mm}$ in diameter and approximately $250 \mu \mathrm{m}$ in height. As the biofilm grows, it adheres to both the top and bottom plate of the device. The top plate can then be rotated or repositioned vertically and the stress and strain induced in the biofilm can be monitored. These measurements can then be used to infer material properties of the biofilm. Using the hrIBM model, we set up a simulation to reproduce experiments described in [26].

In order to reproduce the biofilm in simulation, 3D position data sets obtained by high resolution microscopy of live biofilms are used to initialize the positions of bacteria in the computational domain. The experimental setup used to obtain these data sets is described in [26, 25] and [30]. Although the biofilm position data sets that we use, which were obtained from the experiments described in [25], are not the ones grown and tested in the bioreactor, they are from biofilms grown under similar physical and nutrient availability conditions. A key result seen from our simulations is that the material properties computed by our model of the different data sets are similar to each other. This indicates that the material properties obtained through simulation must depend on larger scale structural properties of the biofilm and may be treated as bulk material properties of the biofilm. For validation we compared bulk properties computed by our model to those measured in experiments. The methods used to compute these quantities are discussed in the next subsections.

In [26], small amplitude rheometry (SAR) is used to characterize the viscoelastic behavior of S. epidermidis biofilms. In SAR experiments, the upper plate of the rheometer is rotated to induce a sinusoidal shear deformation such that the average strain amplitude at the top of the biofilm is a fixed value and the corresponding stress is measured. In [26], the strain amplitude was set to 0.13 at the outer radius of the rheometer since this strain amplitude is found to be in a regime of primarily linear and elastic mechanical behavior. In Figure (4), we show images of the rheometer setup and an illustration of the SAR and compliance experiments.

Creep compliance testing is another characterization technique used in [26] and also in [29] to characterize biofilms. In a creep compliance test, a constant shear stress is applied to the biofilm through the top plate of the rheometer. This induces a time dependent strain which can be measured.

We will now heuristically show that for a small, box-shaped sample of the biofilm that is not near the rotational center or the outer edge of the rheometer, the effects of the rheometer's cylindrical geometry are negligible Thus, the rotational motion that occurs during SAR tests can be well approximated as linear shear. This assumption is pragmatic as it greatly reduces the computational expense our simulations and simplifies the discretization process. 


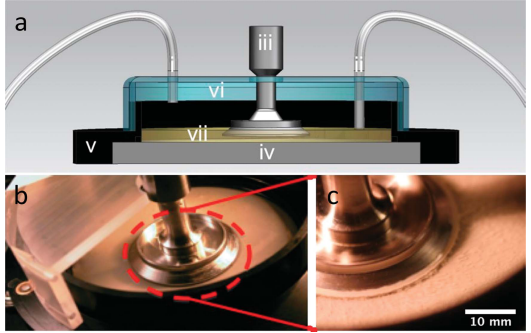

(a)
Small Amplitude Rheology

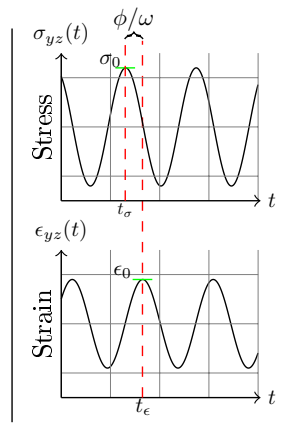

Shear Compliance

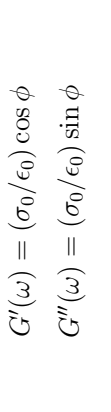

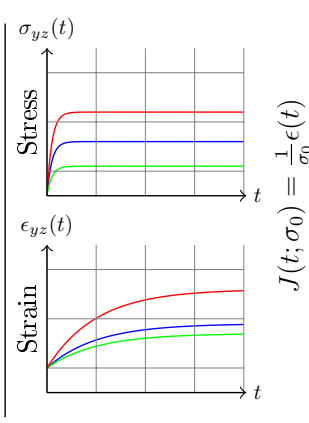

(b)

Figure 4: a) A depiction of the experimental rheometer setup. Reproduced from [26] with permission from the Royal Society of Chemistry. b) An image highlighting the shear rotation of the rheometer. The typical stress and strain profiles expected from SAR and Compliance experiments are shown on the right.

If we temporarily approximate the biofilm as a homogenous, isotropic material, then for low amplitude rotational shear oscillations, the spatial variation in the shear stress and strain will be proportional to the radial distance from the center of the rheometer [10]. If we were to simulate a region of biofilm with dimensions $30 \times 30 \times 30 \mu \mathrm{m}^{3}$ positioned approximately half way between the center and the outer boundary of the rheometer, the ratio of shear strain and shear stress exerted at the inner and outer boundaries of the simulated section of biofilm is greater than 0.99. Thus the deviation from linear shear is minimal in the computational domain. Of course, biofilms are heterogeneous and may be anisotropic however, the previous rough calculation indicates that rectangular geometry and linear shear should provide an accurate approximation of the motion of the biofilm.

\subsection{Computation of Rheological Properties}

In order to compute the desired dynamic moduli, and compliance modulus results, the stress and strain experienced by the biofilm during simulation must be computed.

The stress $\boldsymbol{\sigma}$, is computed as the sum of stress due to the fluid motion, $\boldsymbol{\sigma}^{f}$, and stress induced by the straining of inter-bacteria connections within the biofilm $\sigma^{b}$. Although each component of stress is computed separately during simulations, distinct simulations cannot be used to individually test $\boldsymbol{\sigma}^{b}$ and $\boldsymbol{\sigma}^{f}$ since they are coupled.

In order to calculate the strain $\epsilon$, a set of tracer particles is tracked throughout the simulation. Spatial derivatives can then be calculated to obtain approximations of the strain. Additionally, since only small amplitude strains are observed, the linear relation, $\boldsymbol{\epsilon}=\frac{1}{2}\left(\nabla \mathbf{d}+\nabla^{T} \mathbf{d}\right)$ is an accurate approximation of the strain for a displacement vector d.

Viscoelastic materials are often characterized through their time dependent stress response to strain or their time dependent strain response to stress. For a general viscoelastic material, given that the stress and strain are sufficiently smooth functions of time, constitutive relations between the stress and strain may be written in terms of a convolution with viscoelasticity tensors as:

$$
\begin{aligned}
\sigma_{i j}(\mathbf{x}, t) & =\int_{-\infty}^{t} G_{i j k l}(\mathbf{x}, t-\tau) \frac{d}{d \tau} \epsilon_{k l}(\mathbf{x}, \tau) d \tau \\
\epsilon_{i j}(\mathbf{x}, t) & =\int_{-\infty}^{t} J_{i j k l}(\mathbf{x}, t-\tau) \frac{d}{d \tau} \sigma_{k l}(\mathbf{x}, \tau) d \tau
\end{aligned}
$$

where $\sigma_{i j}$ is the stress tensor, $\epsilon_{i j}$ is the strain tensor, and $G_{i j k l}$ and $J_{i j k l}$ are fourth order viscoelasticity tensors (see [10], $\S 1$ for a derivation). In the literature, $\boldsymbol{G}$ is often called the relaxation modulus and $\boldsymbol{J}$ is called the compliance modulus. For linear, isotropic materials, the expression for $G_{i j k l}$ simplifies to $G_{i j k l}=\frac{1}{3}\left(G_{1}(t)-G_{2}(t)\right) \delta_{i j} \delta_{k l}+$ $\frac{1}{2} G_{1}\left(\delta_{i k} \delta_{j l}+\delta_{i l} \delta_{j k}\right)$, where $\delta_{m n}$ is the Kronecker delta function and Einstein summation notation is used. The two functions, $G_{1}(t)$ and $G_{2}(t)$ correspond respectively to shear stress and dilatational stress. Analogous expressions exist for the compliance tensor.

Although the viscoelastic moduli are spatially heterogeneous, we believe that more meaningful results are obtained in the mean field, or spatially averaged, time (and frequency) dependent values for $\boldsymbol{\epsilon}, \boldsymbol{\sigma}, \boldsymbol{G}$, and $\boldsymbol{J}$. These quantities depend less on the exact configuration of bacteria in a biofilm and behave more like bulk material parameters that can be measured experimentally. Although the interconnected links used to model the connections between 
adjacent bacteria each individually introduce anisotropy into the model, under the conditions of our simulations, the overall behavior of the biofilm is not highly anisotropic.

As can be seen in Figure 5, fluid flow is primarily in the $z$ direction and the $y$ coordinate indicates height in the simulations. Thus the $\epsilon_{y z}$ and $\sigma_{y z}$ components of the stress and strain must be computed at the top plate in order to determine the shear moduli.

\subsubsection{Computation of Strain}

Although a single phase Newtonian fluid will behave viscously (i.e., the stress only depends on the strain rate, not strain itself), in a biofilm the fluid component is influenced by the elastic components of the biofilm and thus the stress state in a biofilm depends directly on the strain (along with the strain rate). In order to compute the strain, the displacement field must be computed. The displacement, $\mathbf{d}$ of a particle located at $\mathbf{x}_{0}$ at time, $t=0$ in a material undergoing deformation can be found by solving the following ODE:

$$
\frac{\partial}{\partial t} \mathbf{d}\left(\mathbf{x}_{0}, t\right)=\int_{\Omega} \mathbf{u}(\mathbf{x}, t) \delta\left(\mathbf{x}-\mathbf{d}\left(\mathbf{x}_{0}, t\right)-\mathbf{x}_{0}\right) d \mathbf{x} ; \quad \mathbf{d}\left(\mathbf{x}_{0}, 0\right)=\mathbf{0} .
$$

In the biofilm simulations, "tracer" particles with positions denoted by $\mathbf{S}(x, y, z)$, are initialized at heights $y_{L}-\gamma$, $y_{L}-\gamma-h$, and $y_{L}-\gamma-2 h$, near the top of the biofilm at $t=0$. At each time step, the positions of the tracers are updated using the same $\delta$ function interpolation used to update the bacteria positions. With these tracers, the deformation of the biofilm can be tracked throughout the simulation.

In the simulations, the $\epsilon_{y z}$ component of strain is needed at the upper boundary of the domain. Therefore, the tracers are initialized near the top of the domain in three vertically aligned layers. This is done to make the numerical approximation of derivatives of the form $\partial d_{z} / \partial y$ simpler to compute. With the initial arrangement of tracers in vertically aligned layers, the centered finite difference approximation

$$
\epsilon_{y z}(\mathbf{S}, t) \approx \frac{1}{2}\left(\frac{\partial d_{z}}{\partial y}+\frac{\partial d_{y}}{\partial z}\right) \approx \frac{1}{2}\left(\frac{\partial d_{z}}{\partial y}\right) \approx \frac{1}{2}\left(\frac{d_{z}\left(\mathbf{S}(y+h)-d_{z}(\mathbf{S}(y-h))\right.}{2 h}\right)
$$

can be used to approximate the strain. Since the entire upper plate moves at a single velocity at any given time, $\partial d_{y} / \partial z$ is negligible in this case, whereas in general, this term is required to compute the shear strain. For our simulations, the strain was also approximated in terms of material coordinates.

\subsubsection{Computation of Stress Induced by Fluid Motion}

From Newton's viscosity law the $\sigma_{y z}^{f}$ component of stress can be found as

$$
\sigma_{y z}^{f}=\mu(\mathbf{x})\left(\frac{\partial u_{z}}{\partial y}+\frac{\partial u_{y}}{\partial z}\right)
$$

Since the velocity field is already known from solving the Navier-Stokes equations at each time step, the relevant derivatives can be approximated by finite difference approximations. As with the strain calculation, the second term, $\partial u_{y} / \partial z$, is zero since the $y$ velocity on the entire top plate of the rheometer is zero. The reported value of $\sigma_{y z}^{f}$ at each time step is then found by spatially averaging over the top $2.5 \mu \mathrm{m}$ of the domain. This is done instead of just averaging over the very top of the domain in case there are numerical boundary layers in the fluid flow field near the boundary. Although we did not observe any boundary layers, they are known to sometimes arise in projection method based fluid solvers $[6,24] .^{3}$

\subsubsection{Computation of Stress Induced by the Biofilm Configuration}

In order to compute the force exerted by the biofilm connections on the top plate, we integrate the Eulerian force field induced by bacteria adhered to the top plate. To determine if a bacteria is adhered, we choose a distance, $\gamma=0.4 \mu \mathrm{m}$ from the top plate, and assume that each bacteria with $y$ coordinate in the interval $\left[y_{L}-\gamma, y_{L}\right]$ is adhered to the top, and that its $z$-component of velocity is fixed to be that of the upper plate. For these bacteria, any force applied on them by spring-like connections to other bacteria behaves like a force exerted by the biofilm on the upper plate instead of on the bulk fluid. The sum of these forces is used to compute the stress induced by the spring-like connections by means of Cauchy's traction law,

$$
\boldsymbol{\sigma}^{b} \mathbf{n}=\frac{\mathbf{F}^{b}}{A}
$$

\footnotetext{
${ }^{3}$ Boundary layers can also be mitigated through adjustments to the boundary conditions for $\mathbf{u}^{*}$ in conjunction with a Crank-Nicholson time stepping scheme.
} 
The outward unit normal, $\mathbf{n}$, is $(0,1,0)$ in this case since the top plate is parallel to the $x z$ plane. The force, $\mathbf{F}^{b}$ is found by integrating the Eulerian force density field that would be generated by the biofilm nodes adhered to the top plate. Additionally, since we are interested in the applied shear stress, $\sigma_{z y}^{b}$, this can be found as $-\frac{F_{z}^{b}}{A}$. Note that $\gamma$ was chosen arbitrarily, however we observed that with $\gamma=0.7 \mu m$ the results were not significantly different.

\subsection{Shear Moduli, $G^{\prime}$ and $G^{\prime \prime}$}

When a nearly isotropic material is subjected to an oscillatory displacement field with frequency $\nu$, we may write the strain as $\epsilon(t)=\epsilon_{0} e^{-i \nu t}$, where $i$ is the imaginary unit and $\epsilon_{0}$ is the strain amplitude. For cases where the strain is primarily only shear strain equation (13) gives, $\boldsymbol{\sigma}(\nu) \approx G_{1}^{*}(\nu) \boldsymbol{\epsilon}(\nu)$ where $G_{1}^{*}(\nu)$ is the Fourier transform in time of $G_{1}(t)$. In general $G_{1}^{*}(\nu)$ is a complex valued function. Breaking the complex shear modulus into its real and imaginary components, $G_{1}^{*}(\nu)=G^{\prime}(\nu)+i G^{\prime \prime}(\nu)$; and given a strain amplitude $\epsilon_{0}(\nu)$ and stress amplitude $\sigma_{0}(\nu)$ (in Pascals),

$$
G^{\prime}(\nu)=\frac{\sigma_{0}(\nu)}{\epsilon_{0}(\nu)} \cos \delta(\nu), \quad G^{\prime \prime}(\nu)=\frac{\sigma_{0}(\nu)}{\epsilon_{0}(\nu)} \sin \delta(\nu) .
$$

Here, $\delta(\nu)$ is known as the loss angle, measured in radians at frequency $\nu$. In the literature, $G^{\prime}(\nu)$ and $G^{\prime \prime}(\nu)$ are often referred to as the storage and loss moduli. They correspond to the elastic and viscous components of a viscoelastic stress strain relationship.

Taking the domain to be a rectangular solid, oriented as shown in Figure 1, we assume that all fields are periodic in the $x$ and $z$ directions. We use the following boundary conditions:

$$
\left.\frac{\partial P}{\partial y}\right|_{y=0, y_{L}}=0 \quad \mathbf{u}(x, 0, z, t)=0 \quad \mathbf{u}\left(x, y_{L}, z, t\right)=\left(0,0, u_{b}(t)\right) .
$$

Along the top boundary, we set the $z$ velocity to be

$$
u_{b}(t)=\epsilon_{0} \nu c(\nu) \frac{\left(e^{2 \nu t}-1\right)\left(\left(e^{4 \nu t}-1\right) \cos \nu t+8 e^{2 \nu t} \sin \nu t\right)}{\left(1+e^{2 \nu t}\right)^{3}} .
$$

This particular function is chosen since it is continuous, at $t=0, u_{z}=0$, and because it converges to within 0.001 of $\epsilon_{0} \nu c(\nu) \cos \nu t$ within half an oscillation, reducing the amount of time needed to run simulations. Experimentally, the strain amplitude, $\epsilon_{0}$ was set to a fixed value over $\nu$. In order to approximate this in simulation, we had to use a prefactor, $c(\nu)$ that can vary slightly in order to maintain similar strain amplitudes at each frequency we simulated. Slightly different values of $G^{\prime}$ and $G^{\prime \prime}$ can be found with different values of $c(\nu)$, however the change is small as long as $c(\nu)$ does not vary too much. In one test, a variation of 0.4957 in $c(\nu)$ yielded a variation in the moduli of approximately $0.4610 \mathrm{~Pa}$ in $G^{\prime}$ and $0.040 \mathrm{~Pa}$ in $G^{\prime \prime}$ at a frequency of $40 \mathrm{~Hz}$.

To initialize the bacteria positions, we take a $9 \mu m \times 27 \mu m \times 9 \mu m$ subset of a $30 \mu m \times 30 \mu m \times 10 \mu m$ bacteria position data field obtained experimentally. This data is also used in the initialization of the viscosity and density fields present in the biofilm. We believe that setting the internal forces to zero at the start is reasonable since experimental results from SAR under both compression and tension yielded similar results in [26]. In Figure 5, the deformation induced by an oscillatory shearing motion is depicted.

In order to tune our model to the experimental data, we adjusted the biofilm viscosity, $\mu_{b}$, the spring constant, $k_{i j}$ used in Hooke's Law, and the cutoff radius with which we allow any two biofilm nodes to be connected by at time $t=0$. For Maxwell's law, we gain an additional viscoelastic parameter that can be tuned, and with the Zener model, there are two additional parameters. In the simulations, we assume that any bacteria separated by a distance of less than $1.62 \mu \mathrm{m}$ are connected. This number was chosen as it leads to biofilms that appear to have similar connectivity to images of live biofilms, such as in [3].

For a spring connecting two points in space, Hooke's Law can be written as

$$
\mathbf{F}_{i j}=k_{i j} \Lambda_{i j}\left(\mathbf{X}, \mathbf{X}_{0}\right)\left(\mathbf{X}_{i}-\mathbf{X}_{j}\right)
$$

with

$$
\Lambda_{i j}\left(\mathbf{X}, \mathbf{X}_{0}\right)=\frac{\left\|\mathbf{X}_{i}(t)-\mathbf{X}_{j}(t)\right\|-\left\|\mathbf{X}_{i}(0)-\mathbf{X}_{j}(0)\right\|}{\left\|\mathbf{X}_{i}(t)-\mathbf{X}_{j}(t)\right\|}
$$

Following [19], we choose each $k_{i j}$ to be a force constant, $F_{s 1}$ divided by the initial separation of bacteria $i$ and $j$. With Maxwell's law and the Zener model, we use an integral form of the standard viscoelastic relation and, integrate by parts to obtain a formula for $\mathbf{F}$. This is done since integration by parts obviates the need to compute any time derivatives of stress or strain. We then approximate the integral at each time step using the trapezoid method. Since the immersed boundary method requires a Lagrangian force density, we divide the values of $\mathbf{F}_{i j}$ 

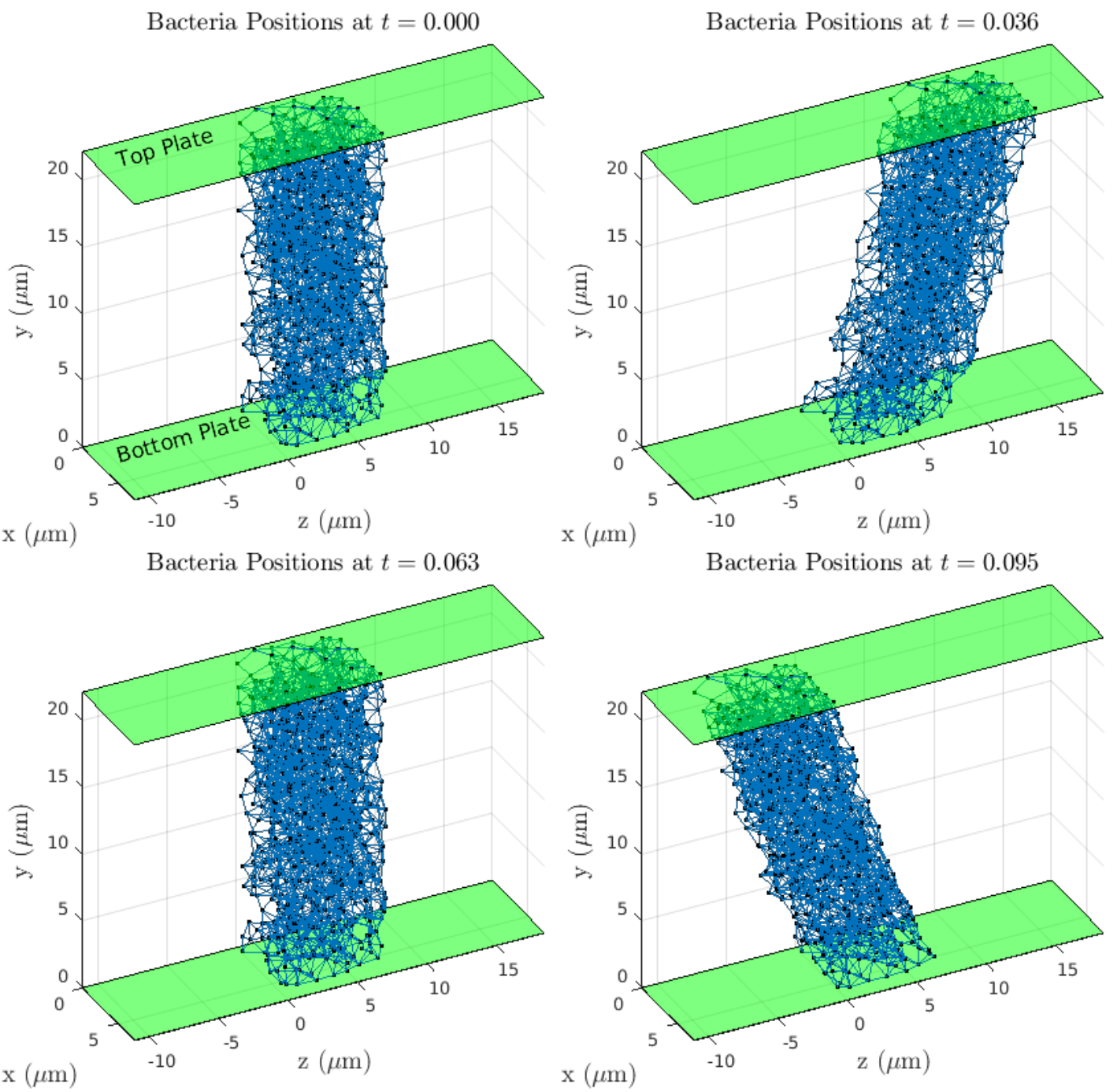

Figure 5: Starting at $t=0$ on the left, the images show how the biofilm is moved as the top plate oscillates. Dots are bacteria locations and lines indicate viscoelastic connections. In the simulations the domain is periodic in the $x$ and $z$ directions. The periodicity is not shown here since it makes it difficult to visualize the effect of deformation on the biofilm. 


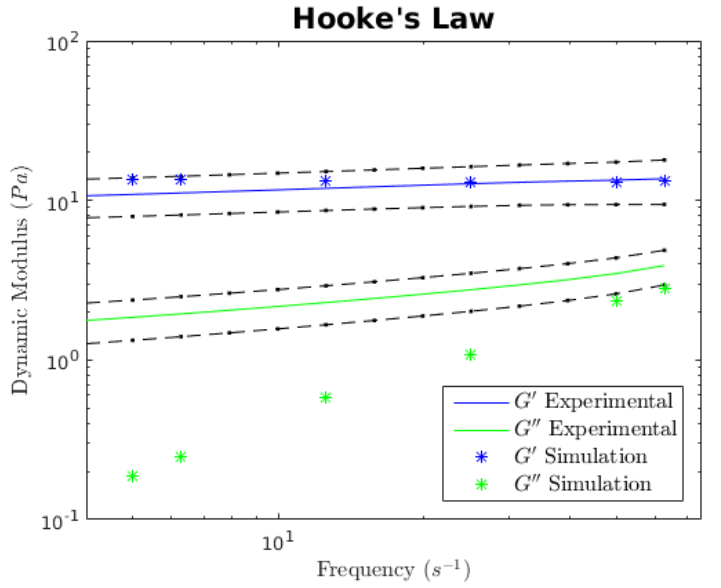

(a)

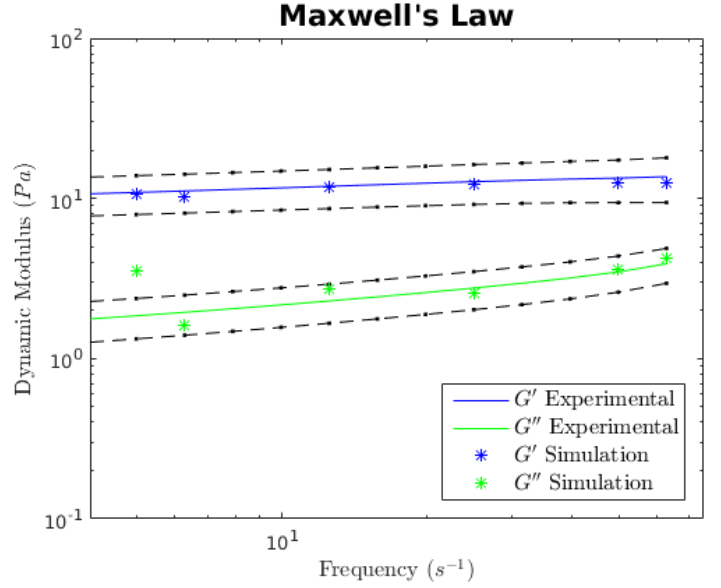

(b)

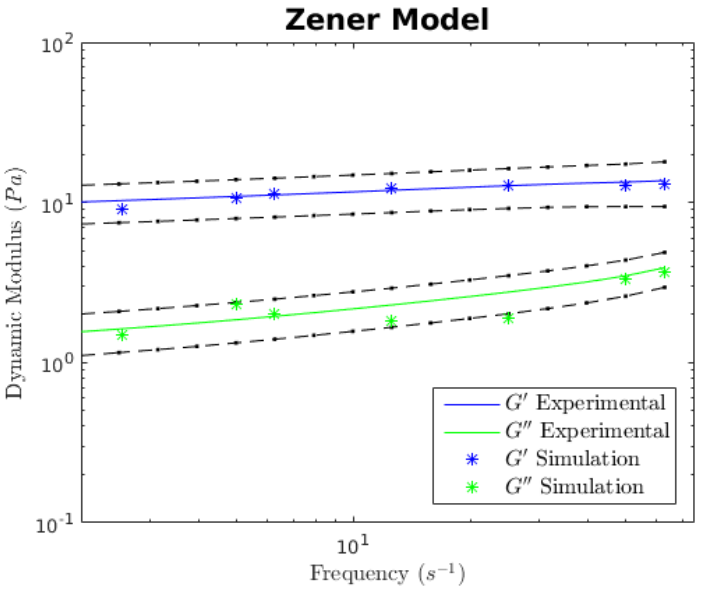

(c)

Figure 6: Comparison between experimentally measured results for $G^{\prime}$ and $G^{\prime \prime}$ and simulated results for three viscoelastic models are depicted. For these results, the force constant was $F_{s 1}=2.30 \cdot 10^{-11} \mathrm{~N}$, the connection distance between bacteria was $1.62 \mu m$, $\mu_{b}=350 \mu_{0}$, and $\rho_{b}=1.12 \rho_{0}$. The dashed lines indicate the experimental error ranges. Subfigure (a) uses Hooke's Law, Subfigure (b) uses Maxwell's law, and Subfigure (c) uses the Zener model. For the Maxwell model, a damping coefficient $F_{d 1}=1.03 \cdot 10^{-11} \mathrm{~N} / \mathrm{s}$ was used, and for the Zener model, the spring coefficient was set to $F_{s 1}=1.64 \cdot 10^{-11} \mathrm{~N}$, the damping coefficient was set to $F_{d 1}=0.051 \cdot 10^{-11} \mathrm{~N} / \mathrm{s}$ and, a second spring coefficient of $F_{s 2}=1.64 \cdot 10^{-11} N$ was used.

determined from the viscoelastic force law by the Lagrangian volume element, $d_{0}^{3}$ to obtain an average force density associated with each bacteria.

In previous works [11,28], identical constitutive relations to (22) are derived from the starting point of energy functionals from which the force density is found by taking a Fréchet derivative of the energy functional. Additionally, in [34] and [12], similar viscoelastic force laws are studied in an immersed boundary method setting and in [34] corresponding dynamic shear moduli are computed.

In Figure 6 we depict the frequency dependence of $G^{\prime}$ and $G^{\prime \prime}$ as determined by the hrIBM model, and compare with experimental data. From these results, it is clear that our model fits experimental data on $G^{\prime}$ quite well. For $G^{\prime \prime}$ the fit is not as strong, although we still do see that for the Maxwell and Zener models, most of the results from simulation are within the range of experimental error. We also observe that even with Hooke's Law which is an elastic constitutive relation, the biofilm still behaves viscoelastically (i.e. $G^{\prime \prime} \neq 0$ ) due to its immersion in a viscous fluid.

\subsection{Creep Compliance Measurements $J(t)$}

Creep compliance is a measure of how a material deforms over time in response to an applied stress. In a rheometer, the compliance is measured applying a step change to the shear stress on the upper plate and observing the resultant shear strain. A step change in stress can be written in terms of a Heaviside step function, $H(t)$ as, $\bar{\sigma}(t)=\sigma_{0} H(t)$ where $\sigma_{0}$ is the magnitude of the step change. For a linear isotropic material with $\sigma_{y z}(t)=\bar{\sigma}(t)$, the 


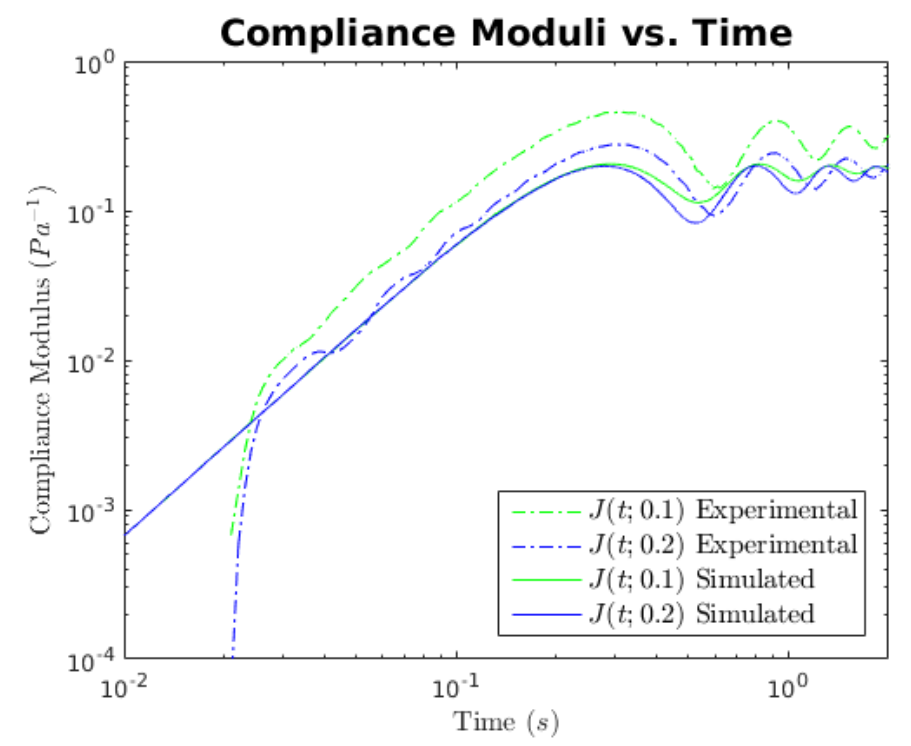

Figure 7: The time dependence of $J\left(t, \sigma_{0}\right)$ is shown above for $\sigma_{0}=0.1 P a$ and $\sigma_{0}=0.2 P a$. The Zener model as used in these simulations. For these levels of applied stress, the change in the compliance modulus is only slight indicating a nearly linear regime.

integral in Equation (14) simplifies to

$$
\epsilon_{y z}(t)=\sigma_{0} J_{1}(t)
$$

From a physical standpoint, most of the bacteria and the bulk of the fluid are only effected by the step change in stress after the stress propagates vertically through the biofilm. However, in the portion of the biofilm adjacent to the upper plate the effect of a change in stress is instantaneous. Thus, we can write a force balance between the forces in the biofilm, the acceleration of the top plate, and the applied force on the top plate. Using this balance of forces, we obtain a time dependent boundary condition for velocity at the top plate. This boundary condition can be written as

$$
\left.\frac{d}{d t} u_{z}\right|_{y=H}=\left(m_{\text {plate }}\right)^{-1}\left(\sigma_{0}-\sigma^{b}-\sigma^{f}\right) A_{\text {plate }}
$$

where $\sigma^{b}$ and $\sigma^{f}$ are the stress exerted by the fluid and the springs in the biofilm at the top plate, $A_{\text {plate }}$ is the area of the upper plate, and $m_{\text {plate }}$ is the mass associated with the top plate of the rheometer. We found that using a mass of $4.22 \cdot 10^{-11} \mathrm{~kg}$ yielded results similar to those seen experimentally.

Numerically, this boundary condition can be written as

$$
\left.u_{z}^{(n+1)}\right|_{y=H}=\left.u_{z}^{(n)}\right|_{y=H}+\left(\frac{A_{\text {plate }} \Delta t}{m_{\text {plate }} u_{0}}\right)\left(\sigma_{0}-\sigma^{b}-\sigma^{f}\right) .
$$

In (26), $\rho_{0}$ and $\mu_{0}$ are the density and viscosity of water, and $L$ is the characteristic length (in this case $10 \mu m$ ). In numerical experiments, rather than immediately impose a step in stress at time 0 , we multiply the applied stress $\sigma_{0}$ by a mollifier, $\left(\frac{2}{1-e^{-\alpha t}}-1\right)$ to mitigate any possible numerical instabilities associated with a discontinuous jump in the wall stress. In this case, $\alpha=155$ is chosen to be large so that the applied stress approaches its equilibrium value within 0.1 seconds. We believe this is reasonable because the very short time scale compliance behavior is not experimentally measurable, and also because a true step change in the stress is not physically realizable. Results from simulations using this boundary condition and two values of $\sigma_{0}$ are shown in Figure 7.

\subsection{Similarity of Material Properties Between Different Bacteria Position Data Sets}

In [13], the spatial statistics of bacteria in a biofilm are studied. We show here that from data sets that have similar spatial distributions of nearest neighbor connections between bacteria, similar bulk property measurements are obtained. In our simulations, we take blocks of experimental data that are $18 \mu \mathrm{m}$ wide, $9 \mu \mathrm{m}$ long, and $27 \mu \mathrm{m}$ high and compute the dynamic moduli of each block at a fixed frequency. The graphs in Figure 8 show the stress and strain of four different biofilms over one period of oscillation.

From Figure 8 and Table 7, we see that in three of the four biofilm data sets, similar results are obtained. We also note that the mean standard error in the experimental results for this particular test was 3.9791 for $G^{\prime}$ and 


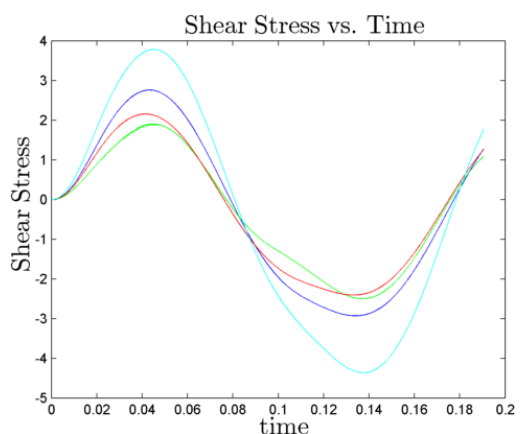

(a)

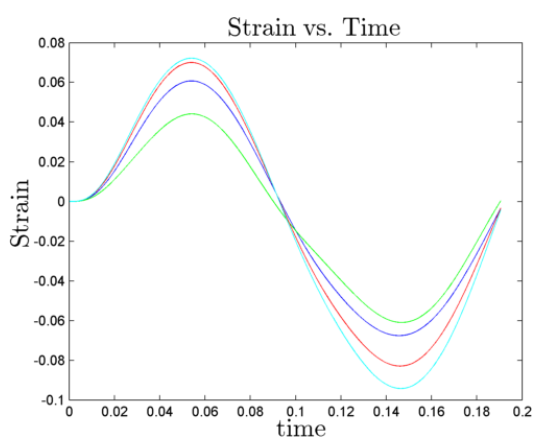

(b)

Figure 8: These graphs show the stress vs. time and strain vs. time for 4 different biofilm samples. The samples are all $18 \mu m \times 27 \mu m \times$ $9 \mu m$ size and contain approximately 2000 bacteria positions.

\begin{tabular}{|c|c|c|c|c|c|}
\hline Simulation & 1 & 2 & 3 & Experimental & Experimental Std. Err. \\
\hline$G^{\prime}$ & 9.03 & 12.71 & 8.17 & 13.399 & 3.9791 \\
\hline$G^{\prime \prime}$ & 2.98 & 3.31 & 2.28 & 3.4812 & 0.8853 \\
\hline
\end{tabular}

Table 7: Results for $G^{\prime}(\nu)$ and $G^{\prime \prime}(\nu)$ are shown at two frequencies for 3 different biofilm coordinate data sets with $\nu=49.91 \mathrm{rad} / \mathrm{s}$. These results show that the physical properties measured here do not depend solely on the exact microstructure of the biofilm, but on some type of more large scale organization of the bacteria positions in space.

0.8836 for $G^{\prime \prime}$. We also suspect that if larger data sets were used, even better agreement would be seen in the computed values of $G^{\prime}$ and $G^{\prime \prime}$.

The importance of this section is in verifying that the properties we are validating can be considered as bulk properties. Since three out of the four data sets provided results within the experimental error deviation, we believe this to be strong evidence the properties we measure are bulk properties.

\subsection{In-Stream Tumbling of Biofilm Fragment}

Bacterial structures exhibit a diverse range of behaviors when subject to fluid flow. One such behavior is the tumbling motion of aggregates in shear flow. To simulate this effect, we conduct simulations in which there are no bacteria anchored along the plates of the domain. Instead, an aggregate of bacteria is located near the middle of the computational domain and the upper and lower plates move in opposite directions as

$$
u_{z}\left(x, y_{L}, z\right)=10^{-3}=-u_{z}(x, 0, z) .
$$

The boundary velocities are scaled by $10^{-3}$ to provide an average shear rate of approximately $114 \mathrm{~s}^{-1}$. This in the range of shear rates examined in [7]. To ensure that the biofilm is not attached to the plates and is sufficiently far from the plate to induce a rotating, or tumbling motion, these simulations only include bacteria that are greater than $8.8 \mu \mathrm{m}$ from either plate at the start. With the physical parameters we use, the bacteria aggregation rotates and is deformed by the fluid shear forces exerted by the fluid [7]. Several snapshots from a simulation of an aggregate tumbling are shown in Figure (9).

In [4], analytical results on the frequency at which a solid ellipsoid will rotate in shear flow are provided. For an ellipsoid with axis aligned with the direction of fluid motion, the rotational frequency is found as

$$
T=\frac{2 \pi\left(a_{1}^{2}+a_{2}^{2}\right)}{a_{1} a_{2} \tau}
$$

where $\tau$ is the shear rate and $a_{1}$ and $a_{2}$ are the principle axes of the ellipse undergoing rotation. In our simulation (see Figure 9), we show that a bacterial aggregate approximated as a hydrodynamically equivalent ellipse will rotate at a frequency similar to the theoretically expected result. The rotational frequency of the aggregate is found by computing the average frequency of rotation of bacteria in the $y z$ plane about the center of mass of the aggregate. In the simulations, we observed a frequency of approximately 0.158 seconds for an aggregate approximated by an ellipse with major axis $a_{1}=2.344 \mu \mathrm{m}$ and first semimajor axis $a_{2}=1.268$, and with shear rate of $114 \mathrm{~s}^{-1}$ containing 110 bacteria. The theoretical result in this case is 0.1317 seconds. For smaller aggregates, the elliptical assumption appears to become less accurate although it still provides results that are on the same order of magnitude. These 

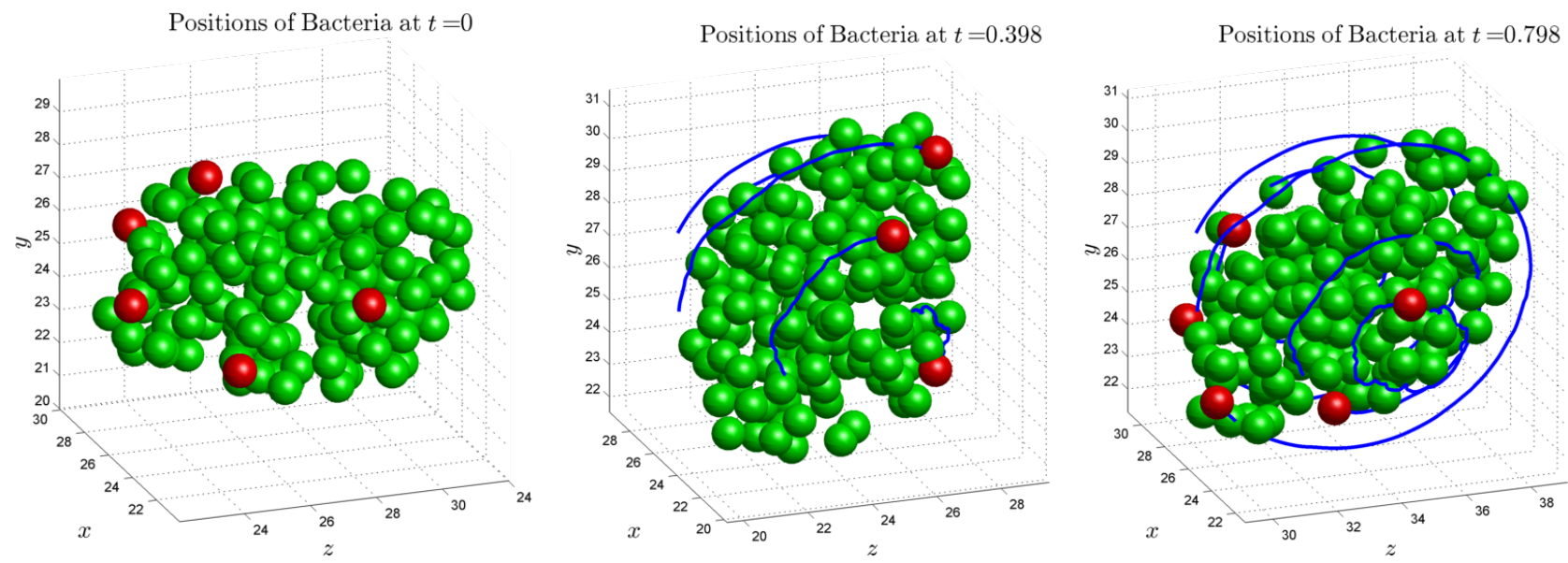

Figure 9: Biofilm aggregate suspended in shear flow rotate over time. Snap shots shown at $0,0.4$ and 0.8 seconds into the simulation. The flattening of the ellipse in response to the shear flow can be distinguished between the first and third figure. Several bacteria are marked red to help show the rotation of the aggregate. The blue lines indicates the trajectories of the marked cells relative to the center of mass of the aggregate. Distances are in micrometers.

\begin{tabular}{|c|c|c|c|c|}
\hline Major axis, $a_{1}$ & First minor axis, $a_{2}$ & Theoretical Period & Observed Period & Relative Error \\
\hline \hline $2.344 \mu \mathrm{m}$ & $1.268 \mu \mathrm{m}$ & $0.1317 \mathrm{~s}$ & $0.1579 \mathrm{~s}$ & $+19.9 \%$ \\
\hline $1.646 \mu \mathrm{m}$ & $1.194 \mu \mathrm{m}$ & $0.1116 \mathrm{~s}$ & $0.1542 \mathrm{~s}$ & $+38.2 \%$ \\
\hline
\end{tabular}

Table 8: Comparison of theoretical and simulated rotational results

results are shown in Table 8. Although we do not currently have experimental results to compare our simulations with, rotational frequency measurement do provide a strong metric for model validation in fields such as red blood cell modeling (see [14]).

\section{Conclusions}

Based on the experimental results shown above and in Part I [19], the heterogeneous rheology Immersed Boundary Method model or hrIBM (Equations (1)-(8)), appears to be an accurate model of the biomechanical response of bacterial biofilms to fluid motion. With the hrIBM model, we resolve biofilms to the scale of individual bacteria. This enables the use of various viscoelastic stress-strain relationships to account for polysaccharide links between bacteria. The spatially heterogeneous ECM also plays a prominent role in the hrIBM model and is treated as a variable viscosity and variable density fluid. We recreate a continuous spatially varying viscosity field, with increased viscosity in the vicinity of the center of mass of each bacteria, by adapting the interpolation procedure found in the immersed boundary method. Although variable viscosity was introduced into the IBM's in [14, 15], this work is the first to consider viscosity as a continuously varying field, not an approximation to a step change in viscosity. Additionally, the authors believe that this work and Part I are the first to consider variable viscosity in an IBM model of biofilms.

In Section 4, a major effort was spent towards validating the hrIBM model by comparing material properties computed by the hrIBM model to experimental data. In particular, we show that the model yields close agreement with experimental results from [26] in which the bacterium S. epidermidis was grown in a bioreactor and characterized using a parallel plate rheometer. Based on the simulations we have conduced it appears that viscoelastic models that contain a Maxwell element provide results comparable to experimental data whereas models that lack a Maxwell element become less accurate at low frequencies. Another development is in showing the similarity between the bulk dynamic moduli over different experimental data sets that possess similar spatial statistics. We also show that suspended aggregates of bacteria in shear flow rotate with a similar period as a hydrodynamically equivalent ellipse. To our knowledge, the hrIBM model is the first that can accurately compute bulk material properties of biofilms based on coupling the microscale connectivity of the biofilm with the heterogeneous rheology of the ECM. 


\section{Future Directions}

Currently the scheme is $\mathcal{O}(\Delta t)$ and $\mathcal{O}(h)$ for the velocity field and bacteria positions. In future work, a predictorcorrector method as used in $[14,15]$, or the Crank-Nicholson time-stepping scheme may be used since, at least in the constant viscosity and density case, these methods can lead to $\mathcal{O}\left(\Delta t^{2}\right)$ convergent Navier-Stokes solvers as shown in [6]. However, even without heterogeneous material properties, obtaining $\mathcal{O}\left(\Delta t^{2}\right)$ convergence in the overall IBM is more complicated and also depends on properties of the discrete Dirac $\delta$ function as discussed in [21, 22].

Modelling and simulation of biofilm detachment is an area of interest in biofilm research. One way to model detachment in our biofilm model would be the inclusion of a stress or strain criterion for when the viscoelastic links in the biofilm should rupture. This approach is explored in [32] where the von Mises stress criterion is used. Another direction would be to follow along the lines of [5], and include model of the reconfiguration of the connectivity of the biofilm over time, especially as the biofilm is deformed due to fluid motion. It is also possible to model the viscoelastic properties of the biofilm through a more complex constitutive model of the Lagrangian force based on the nodal configuration of the bacteria.

Another area that could be explored is the shape of the discrete $\delta$ function used to approximate each bacteria and its associated viscosity halo. It is possible that adjusting this function may allow for more accurate modeling of the mass displacement induced by the bacteria bodies in the bulk fluid. Adjustments to the $\delta$ function may also allow for the inclusion of non-spherical bacteria into the model. It would be interesting to see if similar results are obtained for bacteria that are different shapes.

One possible difficulty in adjusting the discrete $\delta$ function is the preservation of mass in the model. In Equation (2), there is no density dependence as is often seen with the Navier-Stokes equations for a variable density system. For the original IBM, Equation (2) is in fact the correct statement of mass preservation as described in [28]. For the hrIBM, there is an error however, the error is expected to be small in our situation since $\rho(\mathbf{x}, t)$ only varies by $\approx 20 \%$ over the domain (density of bacteria is not highly variable), all simulations are at low Reynold's numbers, and because biofilms are essentially incompressible even though they may have variable density.

\section{Acknowledgements}

This work was supported in part by the National Science Foundation grants PHY-0940991 and DMS-1225878 to DMB, and PHY-0941227 to JGY and MJS, and by the Department of Energy through the Computational Science Graduate Fellowship program, DE-FG02-97ER25308, to JAS. This work utilized the Janus supercomputer, which is supported by the National Science Foundation (award number CNS-0821794), the University of Colorado Boulder, the University of Colorado Denver, and the National Center for Atmospheric Research. The Janus supercomputer is operated by the University of Colorado Boulder. We would also like to thank the reviewers for several useful suggestions that improved this paper.

[1] Alpkvist, E., Klapper, I., 2007-02-22. A Multidimensional Multispecies Continuum Model for Heterogeneous Biofilm Development. Bulletin of Mathematical Biology 69 (2), 765-789.

URL http://link.springer.com/10.1007/s11538-006-9168-7

[2] Alpkvist, E., Picioreanu, C., van Loosdrecht, M. C., Heyden, A., 2006-08-05. Three-dimensional biofilm model with individual cells and continuum EPS matrix. Biotechnology and Bioengineering 94 (5), 961-979.

URL http://doi.wiley.com/10.1002/bit. 20917

[3] Balestrino, D., Ghigo, J.-M., Charbonnel, N., Haagensen, J. A. J., Forestier, C., 2008-03. The characterization of functions involved in the establishment and maturation of klebsiella pneumoniae in vitro biofilm reveals dual roles for surface exopolysaccharides. Environmental Microbiology 10 (3), 685-701.

URL http://doi.wiley.com/10.1111/j.1462-2920.2007.01491.x

[4] Blaser, S., 2002-02. Forces on the surface of small ellipsoidal particles immersed in a linear flow field. Chemical Engineering Science 57 (3), 515-526.

URL http://www.sciencedirect.com/science/article/pii/S000925090100389X

[5] Bottino, D. C., 1998-11. Modeling Viscoelastic Networks and Cell Deformation in the Context of the Immersed Boundary Method. Journal of Computational Physics 147 (1), 86-113.

URL http://linkinghub.elsevier.com/retrieve/pii/S0021999198960740

[6] Brown, D. L., Cortez, R., Minion, M. L., 2001-04. Accurate projection methods for the incompressible navierstokes equations. Journal of Computational Physics 168 (2), 464-499.

URL http://linkinghub.elsevier.com/retrieve/pii/S0021999101967154 
[7] Byrne, E., Dzul, S., Solomon, M., Younger, J., Bortz, D. M., Apr. 2011. Postfragmentation density function for bacterial aggregates in laminar flow. Physical Review E 83 (4), bibtex: Byrne2011.

URL http://link.aps.org/doi/10.1103/PhysRevE.83.041911

[8] Carlslaw, H. S., Jaegar, J. C., 1959. Conduction of Heat in Solids, 2nd Edition. Oxford University Press.

[9] Chen, C., Ren, M., Srinivansan, A., Wang, Q., 2011. 3-d numerical simulations of biofilm flows. East Asian Journal on Applied Mathematics.

URL http://www.csrc.ac.cn/research/publications/201111/w020111117518710826964.pdf

[10] Christensen, R. M., 1982. Theory of Viscoelasticity: An Introduction, 2nd Edition. Academic Press.

[11] Dan Vo, G., Brindle, E., Heys, J., 2010-06. An experimentally validated immersed boundary model of fluidbiofilm interaction. Water Science \& Technology 61 (12), 3033.

URL http://www . iwaponline.com/wst/06112/wst061123033.htm

[12] Dillon, R. H., Fauci, L. J., Omoto, C., Yang, X., 2007-02-15. Fluid dynamic models of flagellar and ciliary beating. Annals of the New York Academy of Sciences 1101 (1), 494-505.

URL http://doi.wiley.com/10.1196/annals.1389.016

[13] Dzul, S. P., Thornton, M. M., Hohne, D. N., Stewart, E. J., Shah, A. A., Bortz, D. M., Solomon, M. J., Younger, J. G., 2011-03-01. Contribution of the klebsiella pneumoniae capsule to bacterial aggregate and biofilm microstructures. Applied and Environmental Microbiology 77 (5), 1777-1782.

URL http://aem.asm.org/content/77/5/1777

[14] Fai, T. G., Griffith, B. E., Mori, Y., Peskin, C. S., 2013-01. Immersed Boundary Method for Variable Viscosity and Variable Density Problems Using Fast Constant-Coefficient Linear Solvers I: Numerical Method and Results. SIAM Journal on Scientific Computing 35 (5), B1132-B1161.

URL http://epubs.siam.org/doi/abs/10.1137/120903038

[15] Fai, T. G., Griffith, B. E., Mori, Y., Peskin, C. S., 2014-01. Immersed Boundary Method for Variable Viscosity and Variable Density Problems Using Fast Constant-Coefficient Linear Solvers II: Theory. SIAM Journal on Scientific Computing 36 (3), B589-B621.

URL http://epubs.siam.org/doi/abs/10.1137/12090304X

[16] Foster, T., 1996. Staphylococcus. In: Baron, S. (Ed.), Medical Microbiology, 4th Edition. University of Texas Medical Branch at Galveston, Galveston (TX).

URL http://www.ncbi.nlm.nih.gov/books/NBK8448/

[17] Gaboriaud, F., Gee, M. L., Strugnell, R., Duval, J. F. L., 2008-10-07. Coupled Electrostatic, Hydrodynamic, and Mechanical Properties of Bacterial Interfaces in Aqueous Media. Langmuir 24 (19), 10988-10995.

URL http://pubs.acs.org/doi/abs/10.1021/la800258n

[18] Ganesan, M., Stewart, E. J., Szafranski, J., Satorius, A. E., Younger, J. G., Solomon, M. J., 2013-05-13. Molar mass, entanglement, and associations of the biofilm polysaccharide of staphylococcus epidermidis. Biomacromolecules 14 (5), 1474-1481.

URL http://pubs .acs.org/doi/abs/10.1021/bm400149a

[19] Hammond, J. F., Stewart, E., Younger, J. G., Solomon, M. J., Bortz, D. M., 2014. Variable Viscosity and Density Biofilm Simulations using an Immersed Boundary Method, Part I: Numerical Scheme and Convergence Results. Computer Modeling in Engineering and Sciences 98 (3), 295-340.

URL http://techscience.com/doi/10.3970/cmes.2014.098.295.html

[20] Lai, M.-C., Peskin, C. S., May 2000. An Immersed Boundary Method with Formal Second-Order Accuracy and Reduced Numerical Viscosity. Journal of Computational Physics 160 (2), 705-719.

URL http://linkinghub.elsevier.com/retrieve/pii/S0021999100964830

[21] Liu, Y., Mori, Y., 2012-01. Properties of Discrete Delta Functions and Local Convergence of the Immersed Boundary Method. SIAM Journal on Numerical Analysis 50 (6), 2986-3015.

URL http://epubs.siam.org/doi/abs/10.1137/110836699 
[22] Liu, Y., Mori, Y., 2014-01. \$L^p $\$$ Convergence of the Immersed Boundary Method for Stationary Stokes Problems. SIAM Journal on Numerical Analysis 52 (1), 496-514.

URL http://epubs.siam.org/doi/abs/10.1137/130911329

[23] Luo, H., Mittal, R., Zheng, X., Bielamowicz, S. A., Walsh, R. J., Hahn, J. K., 2008-11. An immersed-boundary method for flow-structure interaction in biological systems with application to phonation. Journal of Computational Physics 227 (22), 9303-9332.

URL http://linkinghub.elsevier.com/retrieve/pii/S0021999108002623

[24] Orszag, S. A., Israeli, M., Deville, M. O., 1986-03-01. Boundary conditions for incompressible flows. Journal of Scientific Computing 1 (1), 75-111.

URL http://link.springer.com/article/10.1007/BF01061454

[25] Pavlovsky, L., Sturtevant, R. A., Younger, J. G., Solomon, M. J., Feb. 2015. Effects of temperature on the morphological, polymeric, and mechanical properties of Staphylococcus epidermidis bacterial biofilms. Langmuir 31 (6), 2036-2042.

URL http://pubs.acs.org/doi/abs/10.1021/la5044156

[26] Pavlovsky, L., Younger, J. G., Solomon, M. J., 2013. In situ rheology of Staphylococcus epidermidis bacterial biofilms. Soft Matter 9 (1), 122.

URL http://xlink.rsc.org/?DOI=c2sm27005f

[27] Peskin, C. S., 1977-11. Numerical analysis of blood flow in the heart. Journal of Computational Physics 25 (3), $220-252$.

URL http://linkinghub.elsevier.com/retrieve/pii/0021999177901000

[28] Peskin, C. S., 2002-01. The immersed boundary method. Acta Numerica 11.

URL http://www . journals . cambridge .org/abstract_S0962492902000077

[29] Stewart, E. J., Ganesan, M., Younger, J. G., Solomon, M. J., 2015-08-14. Artificial biofilms establish the role of matrix interactions in staphylococcal biofilm assembly and disassembly. Scientific Reports 5, 13081.

URL http://www . nature.com/articles/srep13081

[30] Stewart, E. J., Satorius, A. E., Younger, J. G., Solomon, M. J., Jun. 2013. Role of environmental and antibiotic stress on staphylococcus epidermidis biofilm microstructure. Langmuir 29 (23), 7017-7024.

URL http://pubs.acs.org/doi/abs/10.1021/la401322k

[31] Stull, V. R., 1972-03. Size distribution of bacterial cells. Journal of Bacteriology 109 (3), 1301-1303.

[32] Sudarsan, R., Ghosh, S., Stockie, J. M., Eberl, H. J., 2015-01-28. Simulating biofilm deformation and detachment with the immersed boundary method. arXiv:1501.07221 [physics]ArXiv: 1501.07221.

URL http://arxiv.org/abs/1501.07221

[33] Tierra, G., Pavissich, J. P., Nerenberg, R., Xu, Z., Alber, M. S., 2015-03-25. Multicomponent model of deformation and detachment of a biofilm under fluid flow. Journal of The Royal Society Interface 12 (106), $20150045-20150045$.

URL http://rsif.royalsocietypublishing.org/cgi/doi/10.1098/rsif.2015.0045

[34] Wrobel, J. K., Cortez, R., Fauci, L., Nov. 2014. Modeling viscoelastic networks in Stokes flow. Physics of Fluids 26 (11), 113102.

URL http://scitation.aip.org/content/aip/journal/pof2/26/11/10.1063/1.4900941

[35] Zhang, T., Cogan, N. G., Wang, Q., 2008-01. Phase field models for biofilms. i. theory and one-dimensional simulations. SIAM Journal on Applied Mathematics 69 (3), 641-669.

URL http://epubs.siam.org/doi/abs/10.1137/070691966

[36] Zhang, T., Cogan, N. G., Wang, Q., 2008-07. Phase-field models for biofilms II. 2-d numerical simulations of biofilm-flow interaction. Communications in Computational Physics 4 (1), 72-101.

URL http://www.global-sci.com/freedownload/v4_72.pdf

[37] Zhao, J., Shen, Y., Haapasalo, M., Wang, Z., Wang, Q., 2016-03. A 3d numerical study of antimicrobial persistence in heterogeneous multi-species biofilms. Journal of Theoretical Biology 392, 83-98.

URL http://linkinghub.elsevier.com/retrieve/pii/S0022519315005597 
[38] Zhu, L., Peskin, C. S., 2003. Interaction of two flapping filaments in a flowing soap film. Physics of Fluids $15(7), 1954$.

URL http://scitation.aip.org/content/aip/journal/pof2/15/7/10.1063/1.1582476

[39] Zhuo, J., Dillon, R., 2010-12. Using the immersed boundary method to model complex fluids-structure interaction in sperm motility. Discrete and Continuous Dynamical Systems - Series B 15 (2), 343-355.

URL http: //www . aimsciences.org/journals/displayArticles.jsp?paper ID=5783 